\title{
A Catalytic, Three-Component Assembly Reaction for the Synthesis of Pyrrolidines
}

\author{
Chris V. Galliford, Melissa A. Beenen, SonBinh T. Nguyen, and Karl A. Scheidt* \\ Department of Chemistry, Northwestern University, 2145 Sheridan Road, Evanston, IL 60208
}

\section{Supporting Information}

General Information. All reactions were carried out under an atmosphere of argon or nitrogen in flame-dried glassware with magnetic stirring. THF, $\mathrm{Et}_{2} \mathrm{O}, \mathrm{CH}_{2} \mathrm{Cl}_{2}$ and toluene were purified by passage through a bed of activated alumina. ${ }^{1}$ DME was distilled from sodium. Reagents were purified prior to use unless otherwise stated following the guidelines of Perrin and Armarego. ${ }^{2}$ Purification of reaction products was carried out by flash chromatography using EM Reagent silica gel 60 (230-400 mesh). Analytical thin layer chromatography was performed on EM Reagent $0.25 \mathrm{~mm}$ silica gel 60-F plates. Visualization was accomplished with UV light and anisaldehyde, ceric ammonium nitrate stain, or phosphomolybic acid followed by heating. Melting points were obtained on a Melt-temp 3 instrument and are uncorrected. Infrared spectra were recorded on a Perkin Elmer 1600 series FT-IR spectrometer. ${ }^{1} \mathrm{H}-\mathrm{NMR}$ spectra were recorded on a Varian Inova $500(500 \mathrm{MHz})$ or Mercury $400(400 \mathrm{MHz})$ spectrometer and are reported in ppm using solvent as an internal standard $\left(\mathrm{CDCl}_{3}\right.$ at $\left.7.26 \mathrm{ppm}\right)$. Data are reported as (ap = apparent, $\mathrm{s}=$ singlet, $\mathrm{d}=$ doublet, $\mathrm{t}=$ triplet, $\mathrm{q}=$ quartet, $\mathrm{m}=$ multiplet, $\mathrm{b}=$ broad; coupling constant(s) in Hz; integration. Proton-decoupled ${ }^{13} \mathrm{C}-\mathrm{NMR}$ spectra were recorded on a Varian Inova $500(125 \mathrm{MHz})$ or Mercury $400(100 \mathrm{MHz})$ spectrometer and are reported in ppm using solvent as an internal standard $\left(\mathrm{CDCl}_{3}\right.$ at $\left.77.0 \mathrm{ppm}\right)$. Mass spectra data were obtained on either a Thermo-Finnigan MAT900 high resolution mass spectrometer, a VG 70-250SE HRMS or

(1) Pangborn, A. B.; Giardello, M. A.; Grubbs, R. H.; Rosen, R. K.; Timmers, F. J. Organomettalics 1996, 15, 15181520 .

(2) Perrin, D. D. and Armarego, W. L. Pruification of Laboratory Chemicals; 3rd ed., Pergamon Press, Oxford. 1988. 
Thermo-Finnigan LCQ Advantage HPLC-MS in the Northwestern University Analytical Services Laboratory. Single crystal X-ray data were collected using a Bruker SMART-1000 diffractometer equipped with a CCD detector and processed using SAINT-NT from Bruker.

Ethyl diazoacetate (EDA, 2a), $t$-butyl diazoacetate (2b), and dimethyl acetylene dicarboxylate (DMAD, 3a) were purchased from Aldrich Chemical Company and used without purification. Copper(I) trifluoromethanesulfonate was prepared by a modified method of Kochi and coworkers. ${ }^{3}$ Imines were prepared by combining the corresponding aldehyde (freshly distilled), appropriately substituted aniline, and either activated $4 \AA$ molecular sieves or anhydrous magnesium sulfate in dichloromethane. ${ }^{4}$ The reactions were stirred for $16 \mathrm{~h}$, then filtered and concentrated in vacuo. If necessary, the resulting imines were re-crystallized from a suitable organic solvent mixture to afford imines that were $>95 \%$ pure by ${ }^{1} \mathrm{H}$ NMR analysis.

\section{General Experimental Procedure for the Copper(I)-Catalyzed Reaction:}

To a solution of imine $(1.5 \mathrm{mmol})$ and dipolarophile $(0.5 \mathrm{mmol})$ in $\mathrm{CH}_{2} \mathrm{Cl}_{2}(5.0 \mathrm{~mL})$ heated at reflux was added a solution of a diazo ester $(1.5 \mathrm{mmol})$ in $\mathrm{CH}_{2} \mathrm{Cl}_{2}$ via syringe pump over $3 \mathrm{~h}$. After complete addition, the reaction was heated for an additional hour, cooled and then filtered through silica gel using $\mathrm{CH}_{2} \mathrm{Cl}_{2}(50 \mathrm{~mL})$. The solvent was removed and diastereoselectivities were assigned by analysis of ${ }^{1} \mathrm{H}$ NMR (500 MHz) spectra and correlated with GC/MS integrations. The reaction mixture was purified by flash column chromatograph (ether/hexanes mixtures as eluent), usually by dry loading the sample, to afford the desired cycloaddtion product.

(3) Salomon, R. G.; Kochi, J. K. J. Am. Chem. Soc. 1973, 95, 1889-1897.

(4) (a) Antilla, J. C.; Wulff, W. D. J. Am. Chem. Soc. 1999, 121, 5099-5100. (b) Derdau, V.; Snieckus, V. J. Org. Chem. 2001, 66, 1992-1998. (c) Casarrubios, L.; Perez, J. A.; Brookhart, M.; Templeton, J. L. J. Org. Chem. 1996, $61,8358-8359$. 


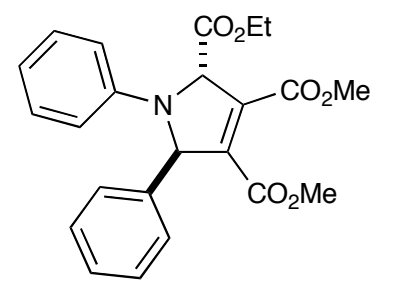

Analytical data for 4a: $\mathrm{R}_{f}=0.3\left(50 \% \mathrm{Et}_{2} \mathrm{O} /\right.$ hexanes); IR (film) 3054, 2987, 1740, 1422, 1265, 896, 740, $705 \mathrm{~cm}^{-1}$; ${ }^{1} \mathrm{H}$ NMR (400 MHz, $\left.\mathrm{CDCl}_{3}\right) \square 7.41-7.14(\mathrm{~m}, 7 \mathrm{H}), 7.10-6.95(\mathrm{~m}, 3 \mathrm{H}), 6.71(\mathrm{~m}, 1 \mathrm{H}), 6.55(\mathrm{~m}$, 2H), $6.03(\mathrm{~d}, J=7.6 \mathrm{~Hz}, 1 \mathrm{H}), 5.77(\mathrm{~d}, J=7.6 \mathrm{~Hz}, 1 \mathrm{H}), 4.14(\mathrm{~m}, 3 \mathrm{H})$, $3.80(\mathrm{~m}, 3 \mathrm{H}), 3.65$ (m, 3H), 1.09 (m, 3H); ${ }^{13} \mathrm{C}$ NMR (100 MHz, CDCl $) \square$ 169.8, 164.1, 150.9, 144.1, 143.6, 138.1, 130.1, 129.2, 129.1, 128.6, 127.5, 118.5, 114.1, 72.1, 69.8, 61.8, 53.5, 53.3, 14.4. LRMS (electrospray): 432, 410, 232, 213, 149, 130; Mass calcd for $\mathrm{C}_{23} \mathrm{H}_{23} \mathrm{NO}_{6}[\mathrm{M}]^{+}$, 409.43. Found 410.

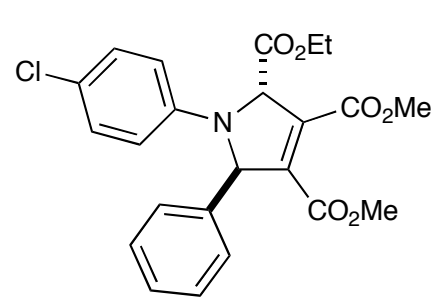

Analytical data for 5: $\mathrm{R}_{f}=0.3\left(50 \% \mathrm{Et}_{2} \mathrm{O} /\right.$ hexanes $) ; \mathrm{mp}=158-159$ ${ }^{\circ} \mathrm{C}$; IR (film) 2985, 2954, 2848, 1738, 1599, 1497, 1437, 1348, 1275, 1242, 1193, 1096, 1028, $817 \mathrm{~cm}^{-1}$; ${ }^{1} \mathrm{H}$ NMR $(500 \mathrm{MHz}$, $\left.\mathrm{CDCl}_{3}\right) \square 7.35-7.22(\mathrm{~m}, 5 \mathrm{H}), 7.05(\mathrm{~m}, 1 \mathrm{H}), 6.50(\mathrm{~m}, 2 \mathrm{H}), 6.01(\mathrm{~d}, J$ $=6.2 \mathrm{~Hz}, 1 \mathrm{H}), 5.25(\mathrm{~d}, J=6.2 \mathrm{~Hz}, 1 \mathrm{H}), 4.24-4.16(2 \mathrm{H}, \mathrm{m}), 3.84(\mathrm{~s}$, 3H), $3.64(\mathrm{~s}, 3 \mathrm{H}), 1.16(\mathrm{~m}, 3 \mathrm{H}) ;{ }^{13} \mathrm{C} \mathrm{NMR}\left(125 \mathrm{MHz}, \mathrm{CDCl}_{3}\right) \square$ 169.9, 162.2, 162.4, 143.8, 142.9, 137.6, 129.7, 129.5, 128.0, 115.3, 71.3, 69.9, 62.2, 52.8, 52.6, 14.3; LRMS (electrospray): 466, 444, 365, 357, 337, 232, 149; Mass calcd for $\mathrm{C}_{23} \mathrm{H}_{22} \mathrm{NO}_{6} \mathrm{Cl}[\mathrm{M}]^{+}, 443.88$. Found 444 .

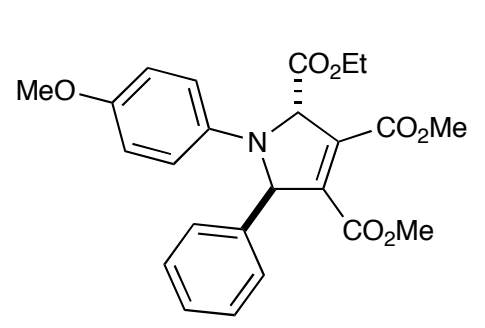

Analytical data for 6: $\mathrm{R}_{f}=0.3\left(50 \% \mathrm{Et}_{2} \mathrm{O} /\right.$ hexanes $) ; \mathrm{mp}=138-141$ ${ }^{\circ} \mathrm{C}$; IR (film) 3054, 2987, 1738, 1513, 1421, 1266, 896, 738, 705 $\mathrm{cm}^{-1} ;{ }^{1} \mathrm{H}$ NMR $\left(500 \mathrm{MHz}, \mathrm{CDCl}_{3}\right) \square 7.41-7.25(\mathrm{~m}, 5 \mathrm{H}), 6.80(\mathrm{~d}, J$ $=9.0 \mathrm{~Hz}, 1 \mathrm{H}), 6.72(\mathrm{~m}, 3 \mathrm{H}), 6.57(\mathrm{~d}, J=9.1 \mathrm{~Hz}, 2 \mathrm{H}), 6.03(\mathrm{~d}, J=$ $7.0 \mathrm{~Hz}, 1 \mathrm{H}), 5.77$ (d, $J=7.3 \mathrm{~Hz}, 1 \mathrm{H}), 4.24-4.16(\mathrm{~m}, 2 \mathrm{H}), 3.89$ (s, $3 \mathrm{H}), 3.78(\mathrm{~m}, 2 \mathrm{H}), 3.68(\mathrm{~s}, 3 \mathrm{H}), 3.62(\mathrm{~s}, 3 \mathrm{H}), 1.17(\mathrm{t}, J=6.9 \mathrm{~Hz}, 3 \mathrm{H}) ;{ }^{13} \mathrm{C} \mathrm{NMR}(125 \mathrm{MHz}$, $\left.\mathrm{CDCl}_{3}\right) \square 170.1,164.0,152.8,138.1,137.8,130.8,129.0,128.3,127.5,116.5,115.3,115.3$, 114.9, 71.5, 70.3, 61.9, 55.8, 53.0, 52.9, 14.3; LRMS (electrospray): 462, 440, 124; Mass calcd for $\mathrm{C}_{24} \mathrm{H}_{25} \mathrm{NO}_{7}[\mathrm{M}+\mathrm{H}]^{+}, 439.36$. Found 440 . 


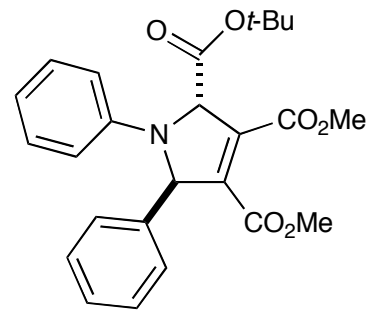

Analytical data for 4b: $\mathrm{R}_{f}=0.38\left(50 \% \mathrm{Et}_{2} \mathrm{O} /\right.$ hexanes $) ; \mathrm{mp}=131-133$ ${ }^{\circ} \mathrm{C}$; IR (film) 2978, 2931, 1732, 1664, 1602, 1503, 1454, 1436, 1348, 1260, 1152, 1104, $1030 \mathrm{~cm}^{-1}$; ${ }^{1} \mathrm{H}$ NMR (500 MHz, $\left.\mathrm{CDCl}_{3}\right) \square 7.40-7.21$ $(\mathrm{m}, 5 \mathrm{H}), 7.10(\mathrm{~m}, 2 \mathrm{H}), 6.75(\mathrm{~m}, 1 \mathrm{H}), 6.55(\mathrm{~m}, 2 \mathrm{H}), 5.99(\mathrm{~d}, J=5.4 \mathrm{~Hz}$, 1H), 5.73 (d, J = 5.4 Hz, 1H), 3.88 (s, 3H), 3.65 (s, 3H), 1.29 (s, 9H); ${ }^{13} \mathrm{C}$ NMR $\left(100 \mathrm{MHz}, \mathrm{CDCl}_{3}\right) \square 178.0,169.0,144.0,144.0,138.5,129.1,128.9,127.1,127.1$, 118.6, 114.1, 71.5, 53.0, 52.6, 28.0. LRMS (electrospray): 460, 438, 382, 360, 279, 149; Mass calcd for $\mathrm{C}_{25} \mathrm{H}_{28} \mathrm{NO}_{6}[\mathrm{M}+\mathrm{H}]^{+}$, 437.48. Found 438 .

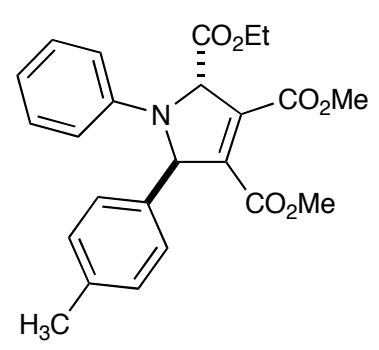

Analytical data for 8: $\mathrm{R}_{f}=0.42\left(50 \% \mathrm{Et}_{2} \mathrm{O} /\right.$ hexanes); IR (film) 3054, 2987, 1739, 1601, 1504, 1438, 1422, 1349, 11265, 1197, 1105, 1025, 896, 737, $705 \mathrm{~cm}^{-1} ;{ }^{1} \mathrm{H} \mathrm{NMR}\left(500 \mathrm{MHz}, \mathrm{CDCl}_{3}\right) \square 7.28-7.09(\mathrm{~m}, 5 \mathrm{H})$, $6.78(\mathrm{~m}, 1 \mathrm{H}), 6.61(\mathrm{~m}, 2 \mathrm{H}), 6.02(\mathrm{~d}, J=6.5 \mathrm{~Hz}, 1 \mathrm{H}), 5.79(\mathrm{~d}, J=6.5$ $\mathrm{Hz}, 1 \mathrm{H}), 4.29-4.12(\mathrm{~m}, 2 \mathrm{H}), 3.86$ (s, 3H), 3.67 (s, 3H), 2.31 (s, 3H), 1.12 (s, 3H); ${ }^{13} \mathrm{C}$ NMR (100 MHz, $\left.\mathrm{CDCl}_{3}\right) \square 170.0,163.5,162.7,144.2,143.7,138.3,1135.1,130.9$, 129.8, 129.2, 127.4, 118.4, 114.1, 71.0, 69.9, 62.0, 52.8, 52.6, 21.5, 14.3. LRMS (electrospray): 446, 424, 387, 213, 149; Mass calcd for $\mathrm{C}_{24} \mathrm{H}_{25} \mathrm{NO}_{6}[\mathrm{M}]^{+}$, 423.46. Found 424.

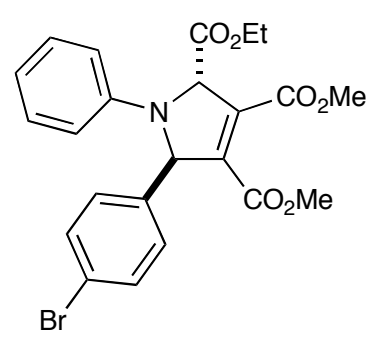

Analytical data for 10: $\mathrm{R}_{f}=0.4\left(50 \% \mathrm{Et}_{2} \mathrm{O} /\right.$ hexanes $) ; \mathrm{mp}=117-119{ }^{\circ} \mathrm{C}$; IR (film) 3050, 3000, 1736, 1664, 1601, 1503, 1436, 1348, 1271, 1245, 1191, 1104, $1010 \mathrm{~cm}^{-1} ;{ }^{1} \mathrm{H}$ NMR (500 $\left.\mathrm{MHz}, \mathrm{CDCl}_{3}\right) \square 7.42(\mathrm{~m}, 2 \mathrm{H})$, $7.21(\mathrm{~m}, 2 \mathrm{H}), 7.11(\mathrm{~m}, 2 \mathrm{H}), 6.23(\mathrm{~m}, 1 \mathrm{H}), 6.52(\mathrm{~m}, 2 \mathrm{H}), 6.00(\mathrm{~d}, J=6.8$ $\mathrm{Hz}, 1 \mathrm{H}), 5.75(\mathrm{~d}, J=6.8 \mathrm{~Hz}, 1 \mathrm{H}), 4.19-4.04(\mathrm{~m}, 2 \mathrm{H}), 3.83(\mathrm{~m}, 3 \mathrm{H}), 3.64$ (m, 3H), 1.09 (m, 3H); ${ }^{13} \mathrm{C}$ NMR (125 MHz, $\left.\mathrm{CDCl}_{3}\right) \square 170.0,163.0,162.3,142.8,142.6,137.2$, $132.0,131.9,129.1,129.0,122.3,118.6,113.9,70.2,69.7,61.8,52.7,52.4,14.0$. LRMS (electrospray): 510, 489, 488, 413, 374, 365, 337, 292, 251, 213, 199, 171, 149, 141; Mass calcd for $\mathrm{C}_{23} \mathrm{H}_{23} \mathrm{NO}_{6} \mathrm{Br}[\mathrm{M}]^{+}$, 488.33. Found 489 . 


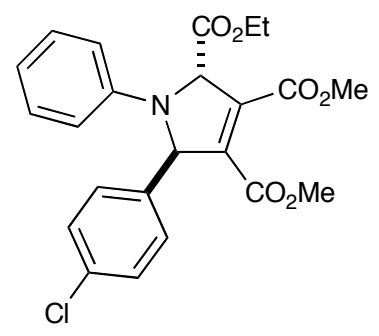

Analytical data for 11: $\mathrm{R}_{f}=0.45\left(50 \% \mathrm{Et}_{2} \mathrm{O} /\right.$ hexanes $) ; \mathrm{mp}=112-115$ ${ }^{\circ} \mathrm{C}$; IR (film) 2985, 2954, 1738, 1664, 1601, 1503, 1437, 1349, 1273, 1245, 1193, 1104, 1014, 751, $692 \mathrm{~cm}^{-1} ;{ }^{1} \mathrm{H} \mathrm{NMR}\left(500 \mathrm{MHz}, \mathrm{CDCl}_{3}\right)$ 7.28-7.20 (m, 3H), $7.05(\mathrm{~m} \mathrm{2H}), 6.75(\mathrm{~m}, 1 \mathrm{H}), 6.50(\mathrm{~m}, 2 \mathrm{H}), 5.95(\mathrm{~d}, J$ $=6.7 \mathrm{~Hz}, 1 \mathrm{H}), 5.75(\mathrm{~d}, J=6.7 \mathrm{~Hz}, 1 \mathrm{H}), 4.20-4.03(\mathrm{~m}, 2 \mathrm{H}), 3.82(\mathrm{~s}, 3 \mathrm{H})$, 3.61 (s, 3H), 1.08 (m, 3H); ${ }^{13} \mathrm{C}$ NMR (125 MHz, $\left.\mathrm{CDCl}_{3}\right) \square 169.8,163.0,162.7,143.7,143.5$, $137.0,134.2,132.1,129.1,129.3,118.8,114.2,70.4,70.0,62.1,53.0,52.7,14.3$. LRMS (electrospray): 466, 444, 391, 232, 213, 149; Mass calcd for $\mathrm{C}_{23} \mathrm{H}_{22} \mathrm{NO}_{6} \mathrm{Cl}[\mathrm{M}]^{+}, 443.88$. Found 444.

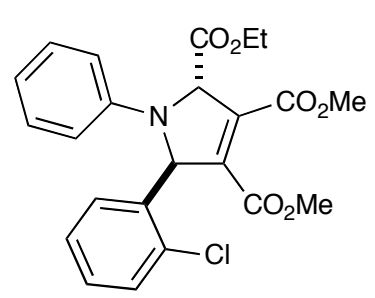

Analytical data for 12: $\mathrm{R}_{f}=0.31\left(50 \% \mathrm{Et}_{2}\right.$ Ohexanes $) ; \mathrm{mp}=112-113{ }^{\circ} \mathrm{C}$; IR (film) 2954, 1741, 1664, 1601, 1503, 1437, 1349, 1274, 1249, 1192, 1104, 1026,751, $691 \mathrm{~cm}^{-1} ;{ }^{1} \mathrm{H}$ NMR (500 MHz, $\left.\mathrm{CDCl}_{3}\right) \square 7.40(\mathrm{~m}, 1 \mathrm{H})$, 7.30-7.09 (m, 5H), $6.75(\mathrm{t}, J=5.8 \mathrm{~Hz}, 1 \mathrm{H}), 6.59(\mathrm{~m}, 3 \mathrm{H}), 5.78(\mathrm{~d}, J=$ $5.8 \mathrm{~Hz}, 1 \mathrm{H}), 4.25-4.11(\mathrm{~m}, 2 \mathrm{H}), 3.91(\mathrm{~m}, 3 \mathrm{H}), 3.68(\mathrm{~m}, 3 \mathrm{H}), 1.13(\mathrm{~m}$, $3 \mathrm{H}) ;{ }^{13} \mathrm{C}$ NMR (125 MHz, $\left.\mathrm{CDCl}_{3}\right) \square 170.1,163.5,162.9,143.9,143.2,135.8,133.8,131.7$, 129.8, 129.4, 128.9, 128.0, 118.8, 113.9, 69.6, 66.8, 62.1, 52.9, 52.7, 14.3. LRMS (electrospray): 466, 444, 232, 149; Mass calcd for $\mathrm{C}_{23} \mathrm{H}_{22} \mathrm{NO}_{6} \mathrm{Cl}[\mathrm{M}]^{+}$, 443.88. Found 444 .

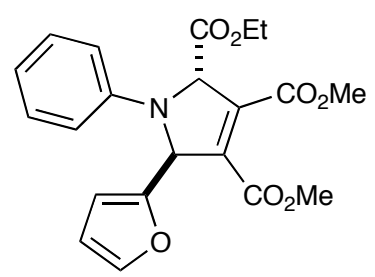

Analytical data for 13: $\mathrm{R}_{f}=0.23\left(50 \% \mathrm{Et}_{2} \mathrm{O} / \mathrm{hexanes}\right) ; \mathrm{mp}=136-138$ ${ }^{\circ} \mathrm{C}$; IR (film) 2954, 2850, 1738, 1668, 1601, 1504, 1437, 1348, 1271, 1245, 1191, 1105, 1013, $750 \mathrm{~cm}^{-1}$; ${ }^{1} \mathrm{H}$ NMR (500 MHz, $\left.\mathrm{CDCl}_{3}\right) \square 7.16$ $(\mathrm{m}, 3 \mathrm{H}), 6.77(\mathrm{~m}, 1 \mathrm{H}), 6.69(\mathrm{~m}, 3 \mathrm{H}), 6.67(\mathrm{~s}, 1 \mathrm{H}), 6.16(\mathrm{~m}, 1 \mathrm{H}), 5.69(\mathrm{~d}$, $J=7.5 \mathrm{~Hz}, 1 \mathrm{H}), 4.19-4.05(\mathrm{~m}, 2 \mathrm{H}), 3.90(\mathrm{~s}, 3 \mathrm{H}), 3.75(\mathrm{~s}, 3 \mathrm{H}), 1.15(\mathrm{~m}, 3 \mathrm{H}) ;{ }^{13} \mathrm{C}$ NMR $(125$ $\left.\mathrm{MHz}, \mathrm{CDCl}_{3}\right) \square 169.5,150.1,144.1,143.0,142.1,139.5,132.6,119.5,114.7,111.0,110.2,79.0$, 64.1, 62.0, 53.9, 53.1, 14.5; LRMS (electrospray): 422, 400, 354, 232, 228, 149, 123; Mass calcd for $\mathrm{C}_{21} \mathrm{H}_{21} \mathrm{NO}_{7}[\mathrm{M}]^{+}, 399.39$. Found 400 . 


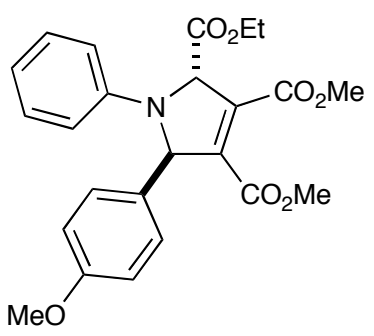

Analytical data for 14: $\mathrm{R}_{f}=0.25\left(50 \% \mathrm{Et}_{2} \mathrm{O} /\right.$ hexanes $) ; \mathrm{mp}=138-140{ }^{\circ} \mathrm{C}$; IR (film) 3055, 2987, 1740, 1437, 1438, 1422, 1265, 896, 740, $705 \mathrm{~cm}^{-1}$; ${ }^{1} \mathrm{H}$ NMR $\left(500 \mathrm{MHz}, \mathrm{CDCl}_{3}\right) \square 7.38-7.25(\mathrm{~m}, 5 \mathrm{H}), 7.11(\mathrm{~m}, 1 \mathrm{H}), 7.06(\mathrm{~m}$, $2 \mathrm{H}), 5.99(\mathrm{~d}, J=6.8 \mathrm{~Hz}, 1 \mathrm{H}), 5.74(\mathrm{~d}, J=6.8 \mathrm{~Hz}, 1 \mathrm{H}), 4.22-4.11(\mathrm{~m}, 2 \mathrm{H})$, $3.82(\mathrm{~s}, 3 \mathrm{H}), 3.62(\mathrm{~s}, 3 \mathrm{H}), 1.15$ (s, 3H); ${ }^{13} \mathrm{C}$ NMR (100 MHz, $\left.\mathrm{CDCl}_{3}\right)$ 169.8, 163.0, 162.4, 143.9, 142.2, 137.7, 131.1, 129.4, 129.2, 128.9, 127.5, 123.6, 116.5, 115.2, 71.3, 69.9, 62.2, 52.9, 52.7, 14.4 LRMS (electrospray): 462, 440, 213, 182, 130, 128; Mass calcd for $\mathrm{C}_{24} \mathrm{H}_{25} \mathrm{NO}_{7}[\mathrm{M}]^{+}, 439.46$. Found 440 .

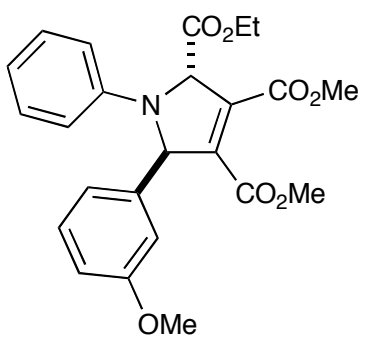

Analytical data for 15: $\mathrm{R}_{f}=0.26\left(50 \% \mathrm{Et}_{2} \mathrm{O} /\right.$ hexanes); IR (film) 3054, 2988, 1740, 1497, 1437, 1421, 1265, 908, 735, $650 \mathrm{~cm}^{-1} ;{ }^{1} \mathrm{H}$ NMR $(500$ $\left.\mathrm{MHz}, \mathrm{CDCl}_{3}\right) \square 7.25-718(\mathrm{~m}, 3 \mathrm{H}), 7.15(\mathrm{~m}, 1 \mathrm{H}), 6.90(\mathrm{~m}, 2 \mathrm{H}), 6.80(\mathrm{~m}$, $1 \mathrm{H}), 6.75(\mathrm{~m}, 1 \mathrm{H}), 6.58(\mathrm{~m}, 2 \mathrm{H}), 6.00(\mathrm{~d}, J=5.8 \mathrm{~Hz}, 1 \mathrm{H}), 5.81(\mathrm{~d}, J=5.8$ $\mathrm{Hz}, 1 \mathrm{H}), 4.26-4.09$ (m, 2H), 3.85 (s, 3H), 3.77 (s, 3H), 3.68 (s, 3H), 1.10 (m, 3H); ${ }^{13} \mathrm{C}$ NMR (125 MHz, $\left.\mathrm{CDCl}_{3}\right) \square 169.9,163.2,162.4,144.5,144.3,140.8,130.9,129.8$, $129.2,119.9,118.5,114.2,114.1,113.8,71.2,69.9,61.9,55.4,52.8,52.6,14.3$. LRMS (electrospray): 462, 440. 316, 212, 149, 135, 123, 121, 119; Mass calcd for $\mathrm{C}_{24} \mathrm{H}_{25} \mathrm{NO}_{7}[\mathrm{M}]^{+}$, 439.46. Found 440.

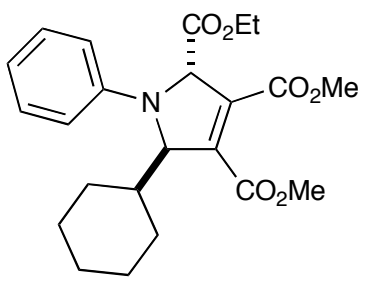

Analytical data for 16: $\mathrm{R}_{f}=0.30\left(50 \% \mathrm{Et}_{2} \mathrm{O} /\right.$ hexanes $) ; \mathrm{mp}=107-108^{\circ} \mathrm{C}$; IR (film) 2933, 2856, 1736, 1601, 1501, $909 \mathrm{~cm}^{-1}$; ${ }^{1} \mathrm{H}$ NMR $(500 \mathrm{MHz}$, $\left.\mathrm{CDCl}_{3}\right) \square 7.25(\mathrm{~m}, 2 \mathrm{H}), 6.79(\mathrm{~m}, 1 \mathrm{H}), 6.68(\mathrm{~m}, 2 \mathrm{H}), 5.50(\mathrm{~d}, J=7.4 \mathrm{~Hz}$, $1 \mathrm{H}), 5.33(\mathrm{~d}, J=7.4 \mathrm{~Hz}, 1 \mathrm{H}), 4.10-3.95(\mathrm{~m}, 2 \mathrm{H}), 3.90$ (s, 3H), 3.81 (s, 3H), $2.00(\mathrm{~m}, 1 \mathrm{H}), 1.80-1.45(\mathrm{~m}, 6 \mathrm{H}), 1.25(\mathrm{~m}, 7 \mathrm{H}) ;{ }^{13} \mathrm{C}$ NMR (125 MHz, $\mathrm{CDCl}_{3}$ ) $\square 169.1$, 165.5, 162.1, 144.6, 131.5, 131.4, 71.8, 70.3, 61.7, 52.7, 52.6, 39.8, 29.4, 27.1, 26.6, 26.4, 14.2. LRMS (electrospray): 438, 417, 416, 260, 228, 186; Mass calcd for $\mathrm{C}_{23} \mathrm{H}_{29} \mathrm{NO}_{6}[\mathrm{M}]^{+}, 415.48$. Found 416. 


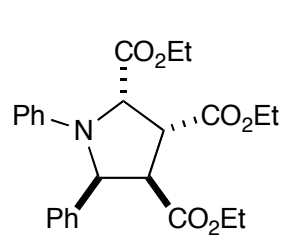

Analytical data for 17: $\mathrm{R}_{f}=0.27$ (40\% $\mathrm{Et}_{2} \mathrm{O} /$ hexanes); IR (film) 2984, 2918, $2850,1727,1629,1602,1503,1300,1266,1030,738 \mathrm{~cm}^{-1} ;{ }^{1} \mathrm{H}$ NMR $(500$ $\left.\mathrm{MHz}, \mathrm{CDCl}_{3}\right) \square 7.27 \square 7.04(\mathrm{~m}, 14 \mathrm{H}), 6.69-6.60(\mathrm{~m}, 4 \mathrm{H}), 6.46(\mathrm{~m}, 2 \mathrm{H}), 5.38$ $(\mathrm{d}, J=4.0 \mathrm{~Hz}, 1 \mathrm{H}), 5.30(\mathrm{~m}, 2 \mathrm{H}), 5.25,(\mathrm{~d}, J=9.1 \mathrm{~Hz}, 1 \mathrm{H}), 4.28-3.96(\mathrm{~m}$, $12 \mathrm{H}), 3.82(\mathrm{~m}, 2 \mathrm{H}), 3.73(\mathrm{~m}, 1 \mathrm{H}), 3.52(\mathrm{~m}, 1 \mathrm{H}), 1.40-0.96(\mathrm{~b}, \mathrm{~m}, 18 \mathrm{H}) ;{ }^{13} \mathrm{C} \mathrm{NMR}(125 \mathrm{MHz}$, $\left.\mathrm{CDCl}_{3}\right) \square 172.3,170.6164 .1,161.8,152.0,148.7,145.4,142.9,142.3,129.5,129.5,129.3$, $129.0128 .9,128.2,127.5,127.3,126.2,125.5,121.3,118.4,118.1,115.2,113.5,112.7,69.0$, 67.0, 64.4, 64.0, 62.8, 61.8, 61.5, 61.2, 55.8, 48.8, 46.6, 16.3, 14.4, 14.4, 14.2, 14.2, 14.1; LRMS (electrospray): 440, 290, 288, 182, 104 Mass calcd for $\mathrm{C}_{25} \mathrm{H}_{29} \mathrm{NO}_{6}[\mathrm{M}]^{+}$, 439.50. Found 440 .

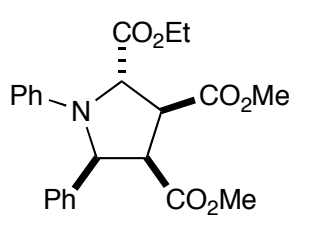

Analytical data for 18: $\mathrm{R}_{f}=0.37$ (40\% $\mathrm{Et}_{2} \mathrm{O} /$ hexanes); IR (film) 2984, 2919, $2850,1727,1629,1601,1503 \mathrm{~cm}^{-1}$; ${ }^{1} \mathrm{H}$ NMR $\left(500 \mathrm{MHz}, \mathrm{CDCl}_{3}\right)$ प7.45प7.25 (b, m, 8H), 7.22-7.14 (m, 6H), 6.85-6.71 (m, 4H), 6.61-6.49 (m, 2H), $5.39(\mathrm{~d}, J=2.5 \mathrm{~Hz}, 1 \mathrm{H}), 5.32(\mathrm{~d}, J=2.5 \mathrm{~Hz}, 1 \mathrm{H}), 5.28(\mathrm{~m}, 1 \mathrm{H}), 5.05(\mathrm{~m}, 1 \mathrm{H}), 4.35-4.28(\mathrm{~m}$, $3 \mathrm{H}), 4.26-4.17(\mathrm{~m}, 5 \mathrm{H}), 4.11-4.04(\mathrm{~m}, 3 \mathrm{H}), 3.85-3.72(\mathrm{~m}, 6 \mathrm{H}), 3.66(\mathrm{~m}, 2 \mathrm{H}), 3.40(\mathrm{~m}, 1 \mathrm{H}), 1.36-$ $1.05 \quad(\mathrm{~m}, \quad 6 \mathrm{H}) ; \quad{ }^{13} \mathrm{C} \quad \mathrm{NMR} \quad\left(125 \quad \mathrm{MHz}, \quad \mathrm{CDCl}_{3}\right)$ ○178.4, 178.2, 172.0, 171.5, 162.1, 162.1, 159.1, 151.3, 130.0, 129.8, 129.6, 129.4, 129.1, 129.0 127.6, 127.4, 126.4, 126.2, 124.2, 123.4, 121.2, 118.6, 115.3, 112.8, 65.7, 64.4, 61.6, 61.3, 58.8, 55.6, 53.8, 53.1, 48.6, 29.9, 14.4, 14.3; LRMS electrospray): 434, 412, 391, 359, 306, 286, 260 , 232, 199, 164; Mass calcd for $\mathrm{C}_{23} \mathrm{H}_{25} \mathrm{NO}_{6}[\mathrm{M}]^{+}, 411.45$. Found 412.

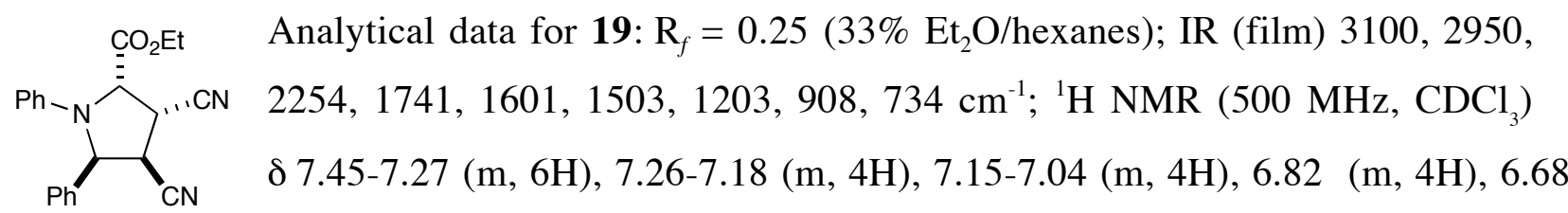
$(\mathrm{m}, 2 \mathrm{H}), 5.35(\mathrm{~d}, J=8.6 \mathrm{~Hz}, 1 \mathrm{H}), 5.18(\mathrm{~d}, J=6.4 \mathrm{~Hz}, 1 \mathrm{H}), 5.12(\mathrm{~d}, J=4.1 \mathrm{~Hz}, 1 \mathrm{H}), 4.96(\mathrm{~d}, J=$ $8.4 \mathrm{~Hz}, 1 \mathrm{H}), 4.36(\mathrm{~m}, 3 \mathrm{H}), 4.13(\mathrm{~m}, 2 \mathrm{H}), 3.87(\mathrm{~m}, 1 \mathrm{H}), 3.73(\mathrm{~m}, 1 \mathrm{H}), 3.41(\mathrm{~m}, 1 \mathrm{H}), 1.38-1.35(\mathrm{~m}$, $3 \mathrm{H}), \quad 1.09-1.05 \quad(\mathrm{~m}, \quad 3 \mathrm{H}) ; \quad{ }^{13} \mathrm{C} \quad \mathrm{NMR} \quad\left(125 \quad \mathrm{MHz}, \quad \mathrm{CDCl}_{3}\right)$ 口169.1, 169.0, 138.0, 136.5, 129.8, 129.7, 129.6, 129.2, 129.2, 126.8, 126.4, 121.4, 119.6, 117.9, 
117.3, 117.0, 116.9, 116.1, 114.8, 113.6, 94.4, 94.4, 67.1, 67.0, 63.2, 63.2, 62.7, 62.1, 42.4, 38.6, 35.0, 33.8, 14.5, 14.4LRMSectrospray): 346, 272, 196; Mass calcd for $\mathrm{C}_{21} \mathrm{H}_{19} \mathrm{~N}_{3} \mathrm{O}_{2}[\mathrm{M}]^{+}$, 345.39. Found 346.

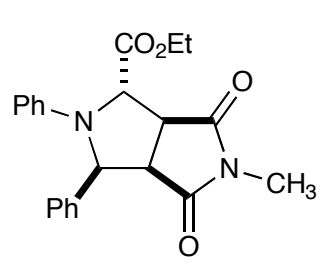

Analytical data for 20: $\mathrm{R}_{f}=0.15\left(50 \% \mathrm{Et}_{2} \mathrm{O} /\right.$ hexanes); IR (film) $\mathrm{cm}^{-1} ;{ }^{1} \mathrm{H}$ NMR (500 MHz, $\left.\mathrm{CDCl}_{3}\right) \square 7.28 \square 7.24(\mathrm{~m}, 4 \mathrm{H}), 7.11$ (m, 2H), 6.77 (m, 2H), $6.65(\mathrm{~m}, 2 \mathrm{H}), 5.41,(\mathrm{~d}, J=4.5 \mathrm{~Hz}, 1 \mathrm{H}), 5.20(\mathrm{~d}, J=10.3 \mathrm{~Hz}, 1 \mathrm{H}), 4.07(\mathrm{~m}$, 2H), $3.98(\mathrm{dd}, J=10.3,4.5 \mathrm{~Hz}, 1 \mathrm{H}), 3.42(\mathrm{dd},=4.1,9.3 \mathrm{~Hz} 1 \mathrm{H}), 3.05-3.00$ $(\mathrm{s}, \quad 3 \mathrm{H}), \quad 1.12 \quad(\mathrm{~m}, \quad 3 \mathrm{H}) ; \quad{ }^{13} \mathrm{C} \quad \mathrm{NMR} \quad\left(125 \quad \mathrm{MHz}, \quad \mathrm{CDCl}_{3}\right)$ $\square 177.2,175.2$, 171.0, 144.9, 141.8, 129.4, 129.2, 127.9, 126.3, 120.1, 117.1, 65.3, 65.0, 61.9, 54. 6, 46.6, 25.5, 14.2 LRMS (electrospray): 401, 379, 305, 182, 149; Mass calcd for $\mathrm{C}_{22} \mathrm{H}_{22} \mathrm{~N}_{2} \mathrm{O}_{4}$ $[\mathrm{M}]^{+}, 378.42$, Found 379 .

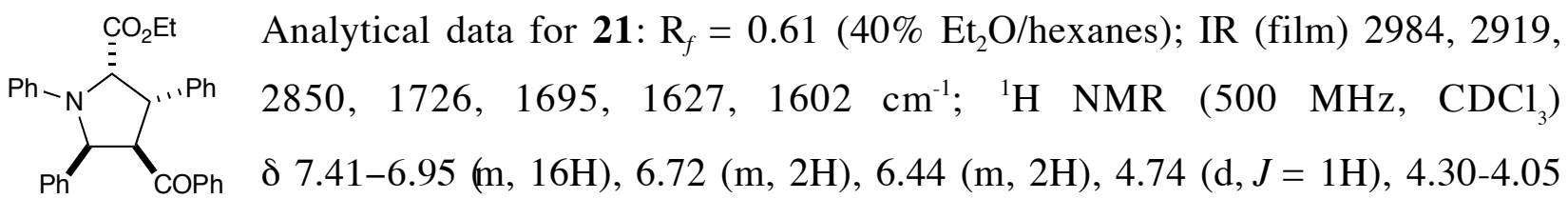
$(\mathrm{m}, 3 \mathrm{H}), 3.52-3.47(\mathrm{dd}, J=13.1 \mathrm{~Hz}, J=9.0 \mathrm{~Hz}, 1 \mathrm{H}), 3.24-3.21(\mathrm{dd}, J=13.1 \mathrm{~Hz}, J=9.0 \mathrm{~Hz}$, $1 \mathrm{H}), \quad 1.12 \quad(\mathrm{~m}, \quad 3 \mathrm{H}) ; \quad{ }^{13} \mathrm{C} \quad \mathrm{NMR} \quad\left(125 \quad \mathrm{MHz}, \quad \mathrm{CDCl}_{3}\right)$ - 207.1, 171.2, 146.2, 144.7, 140.8, 140.5, 131.5, 131.4, 129.1, 128.9, 128.7, 128.6, 128.5, 127.1, $126.7,126.2,119.5,115.1,86.5,82.3,62.6,6.4,54.2,14.4$;

$\square$ ass calcd for $\mathrm{C}_{32} \mathrm{H}_{29} \mathrm{NO}_{3}[\mathrm{M}]^{+}$, 475.58. Found 476 .

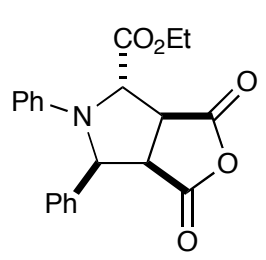

Analytical data for 22: $\mathrm{R}_{f}=0.35$ (45\% Et $2 \mathrm{O} /$ hexanes); IR (film) 2921, 2850, 1718, 1687, 1639, 1278, 1179, $1030 \mathrm{~cm}^{-1}$; ${ }^{1} \mathrm{H} \mathrm{NMR}\left(400 \mathrm{MHz}, \mathrm{CDCl}_{3}\right.$ )

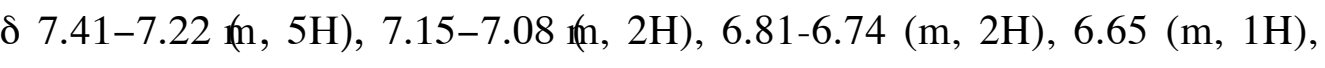
$5.52\left(\mathrm{dd}, \mathrm{J}_{1}=6.5 \mathrm{~Hz}, 6.8 \mathrm{~Hz}, 1 \mathrm{H}\right), 5.45(\mathrm{~d}, J=6.1 \mathrm{~Hz}, 1 \mathrm{H}), 4.21-3.98(\mathrm{~m}, 3 \mathrm{H})$, $3.60,(\mathrm{~m}, \quad 1 \mathrm{H}), \quad 1.12 \quad(\mathrm{~s}, \quad 3 \mathrm{H}) ;{ }^{13} \mathrm{C} \quad \mathrm{NMR} \quad\left(100 \quad \mathrm{MHz}, \quad \mathrm{CDCl}_{3}\right)$ $\square 175.2,173.8,137.6,131.5,129.2$, 129.2, 128.8, 128.6, 126.0, 92.7, 74.4, 65.7, 64.5, 61.8, 49.9, 49.6, 14.4 LRMS (electrospray): 388, 366, 288, 182, 104 Mass calcd for $\mathrm{C}_{21} \mathrm{H}_{19} \mathrm{NO}_{5}[\mathrm{M}]^{+}$, 365.38. Found 366 . 


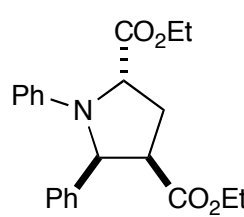

Analytical data for 23: $\mathrm{R}_{f}=0.31$ (45\% Et $2 \mathrm{O} /$ hexanes); IR (film) 2989, 2919, 2850, 1726, 1630, 1602, $1504 \mathrm{~cm}^{-1} ;{ }^{1} \mathrm{H}$ NMR (400 MHz, $\left.\mathrm{CDCl}_{3}\right)$ d 7.29-7.20 (m, 4H), 7.17-7.07 (m, 4H), 6.64-6.54 (m, 2H), 5.27 (d, J = 9.1 Hz, $1 \mathrm{H}), 4.73(\mathrm{~d}, J=8.6 \mathrm{~Hz}, 1 \mathrm{H}), 3.85(\mathrm{~m}, 1 \mathrm{H}), 3.81-3.65(\mathrm{~m}, 4 \mathrm{H}), 2.96-2.84$ (ddd, $J=13.4 \mathrm{~Hz}, 13.4 \mathrm{~Hz}, 9.2 \mathrm{~Hz}, 1 \mathrm{H}), 2.21-2.18\left(\mathrm{dd}, \mathrm{J}_{1}=12.8 \mathrm{~Hz}, 6.1 \mathrm{~Hz}, 1 \mathrm{H}\right), 1.22-1.10$ (m,

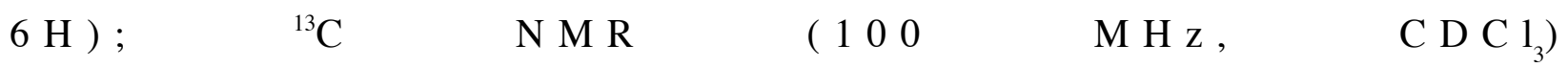
d 139.3, 138.3, 129.1, 128.6, 128.0, 127.3, 117.4, 114.1, 113.6, 113.1, 61.5, 56.9, 39.6, 30.4, 30.1 4.5, 14.2; LRMS (electrospray): Mass calcd for $\mathrm{C}_{22} \mathrm{H}_{25} \mathrm{NO}_{4}[\mathrm{M}]^{+}, 367.44$. Found 368 

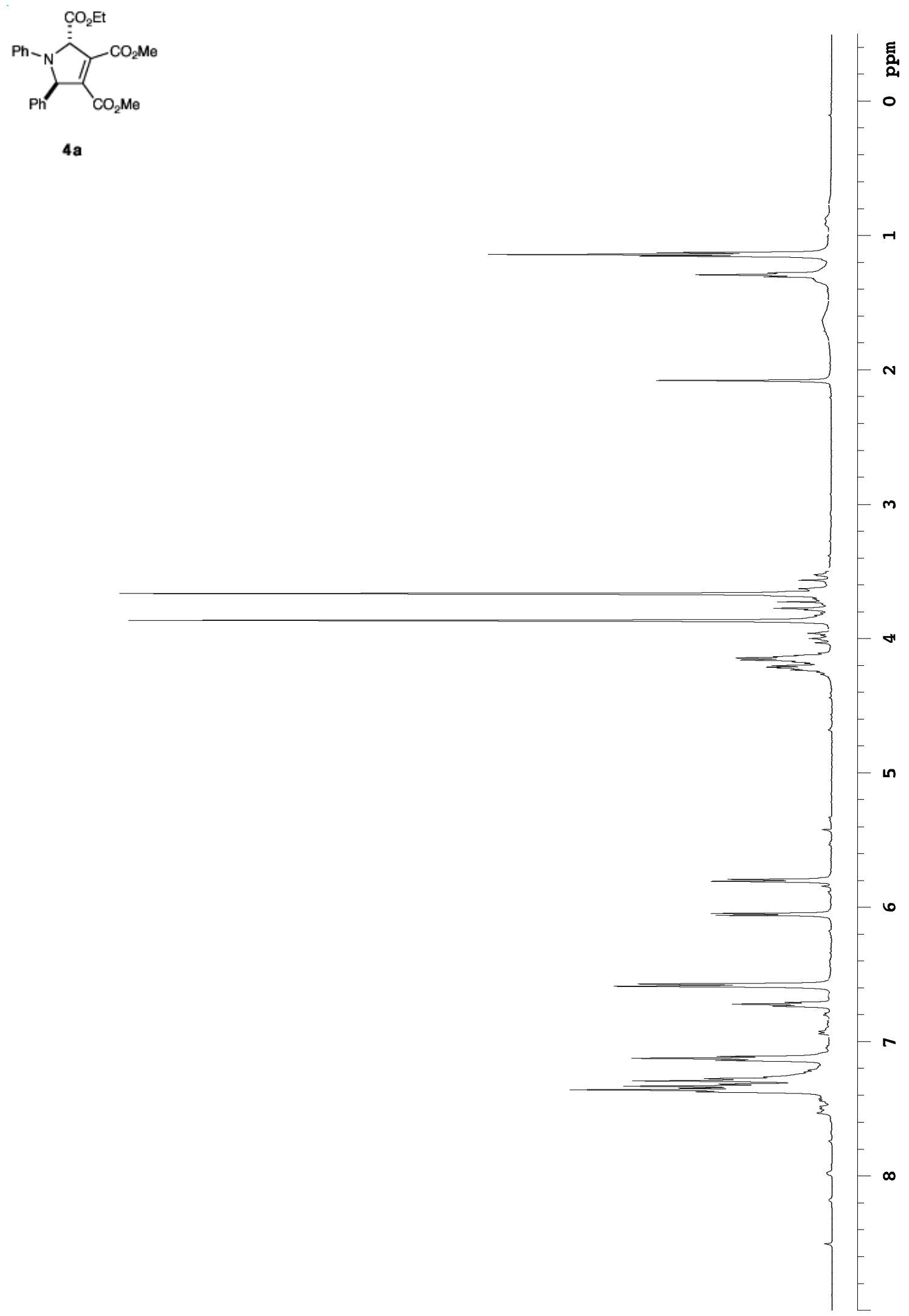

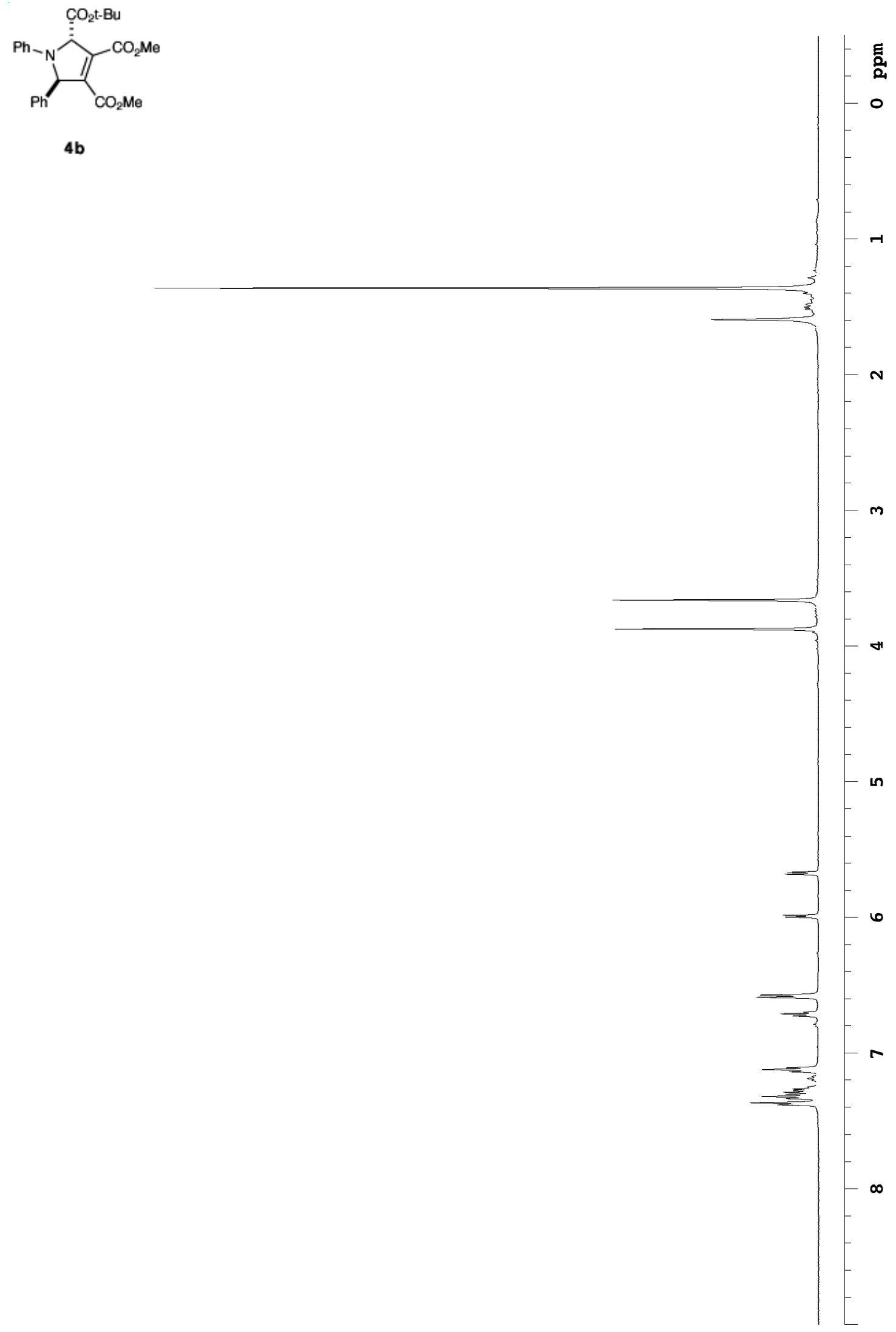


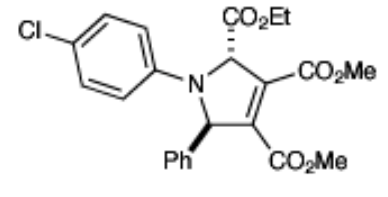

5

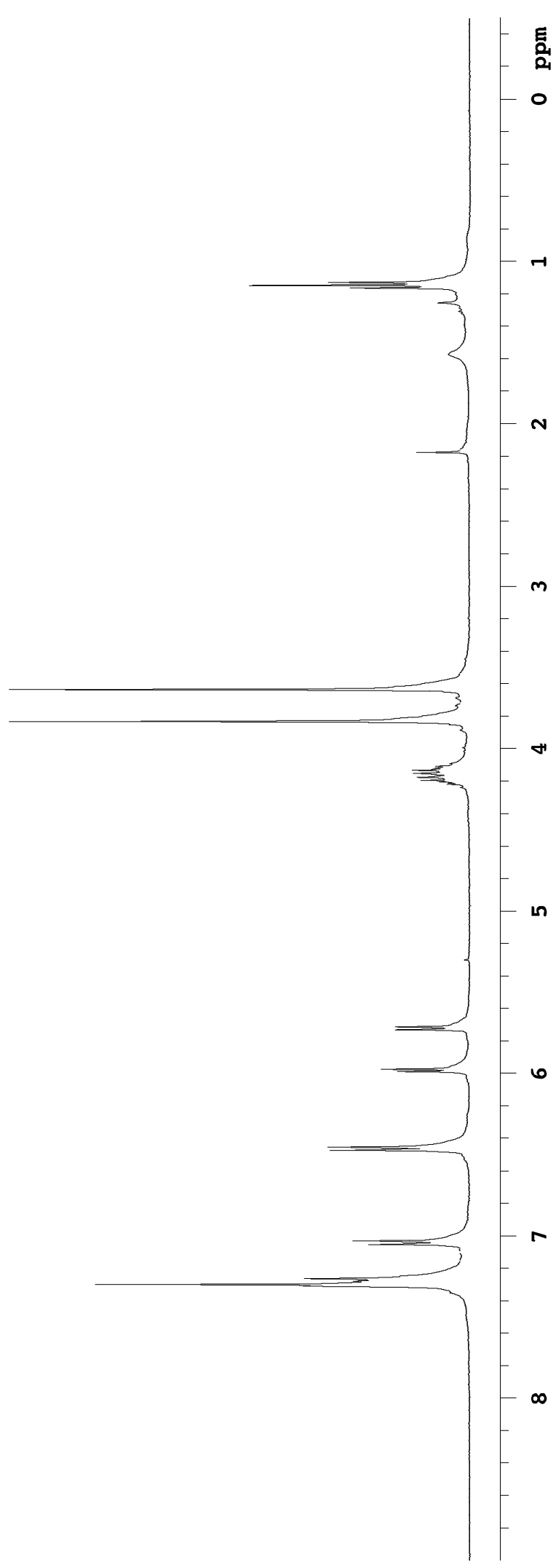



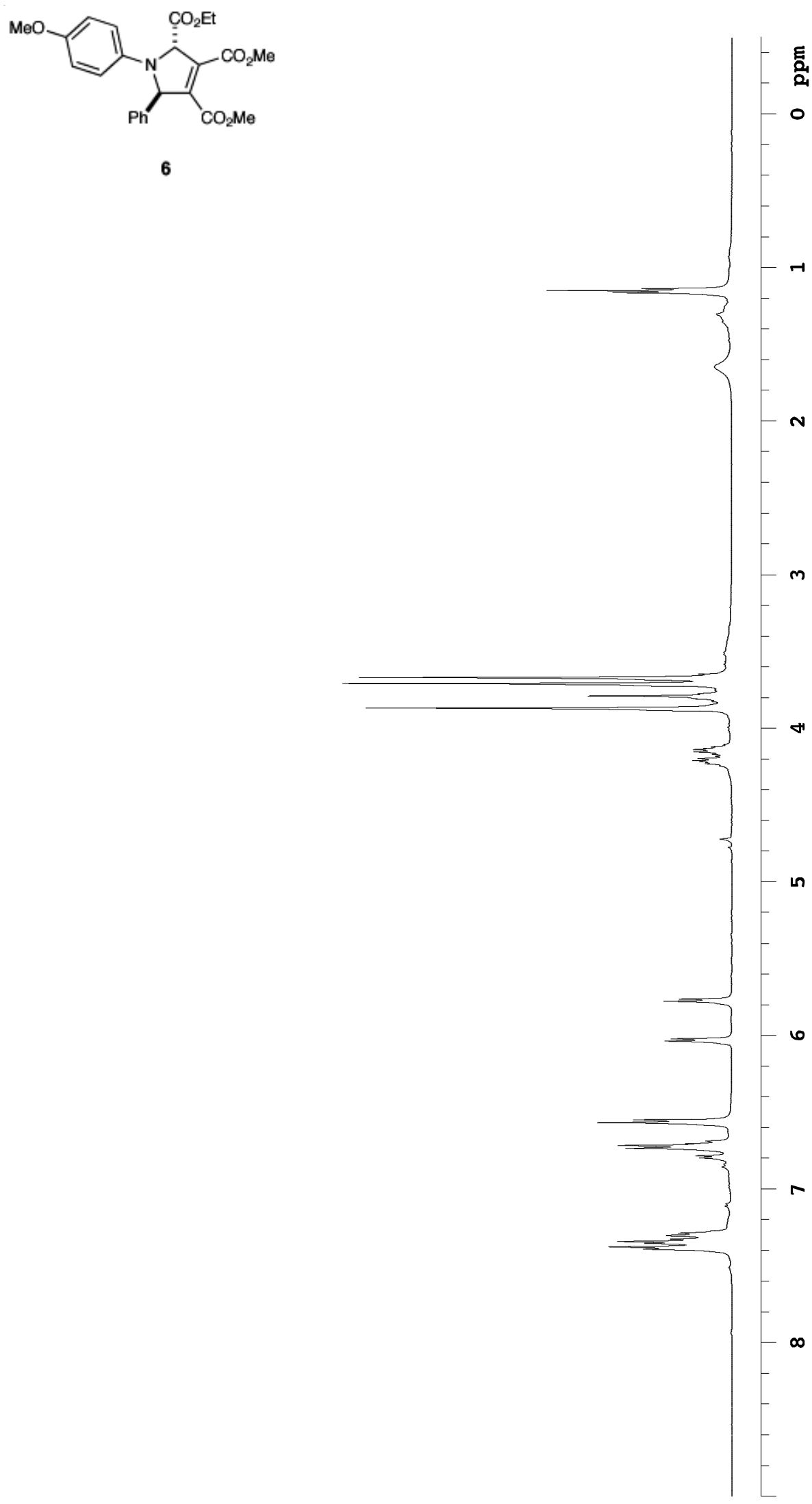

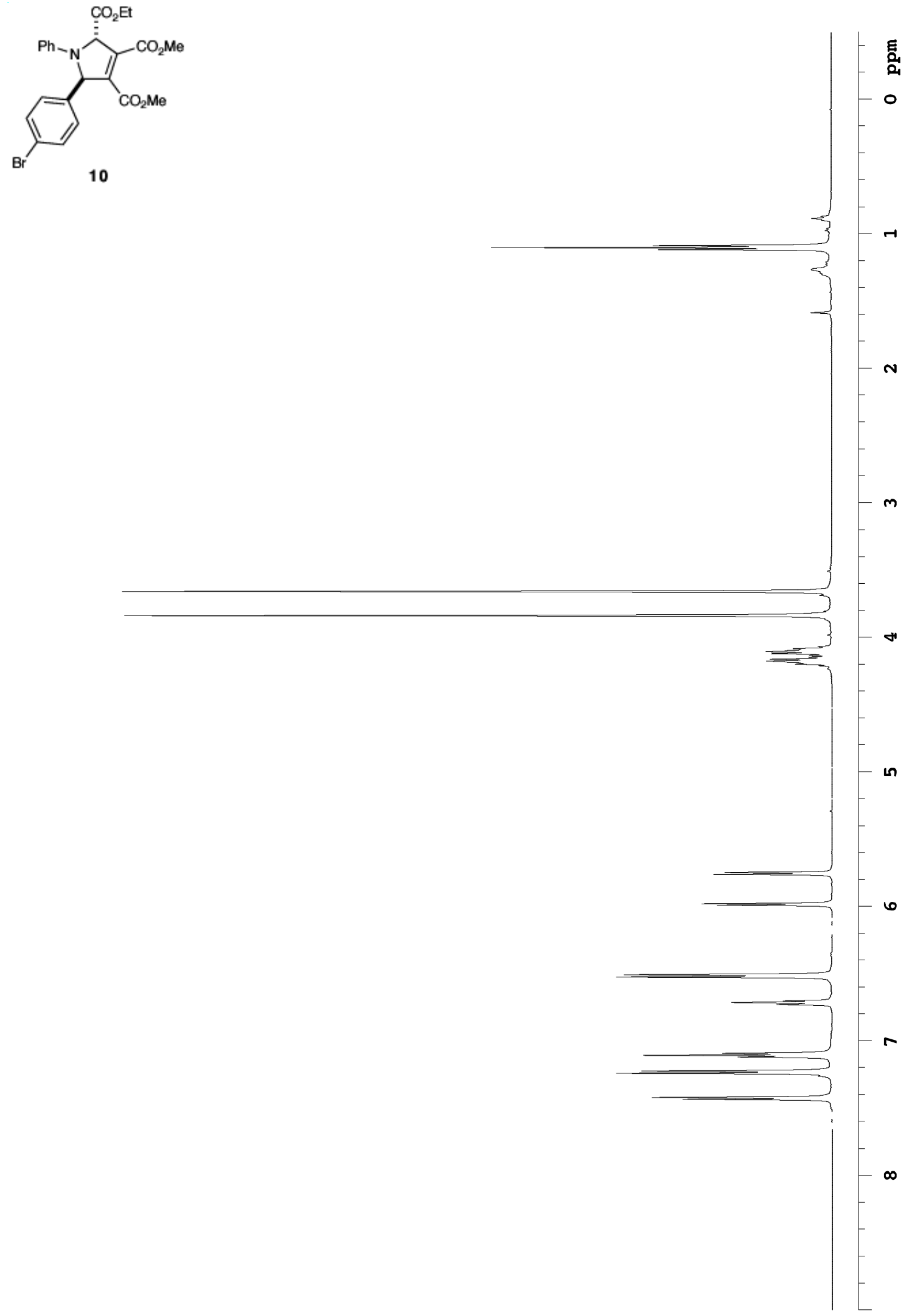

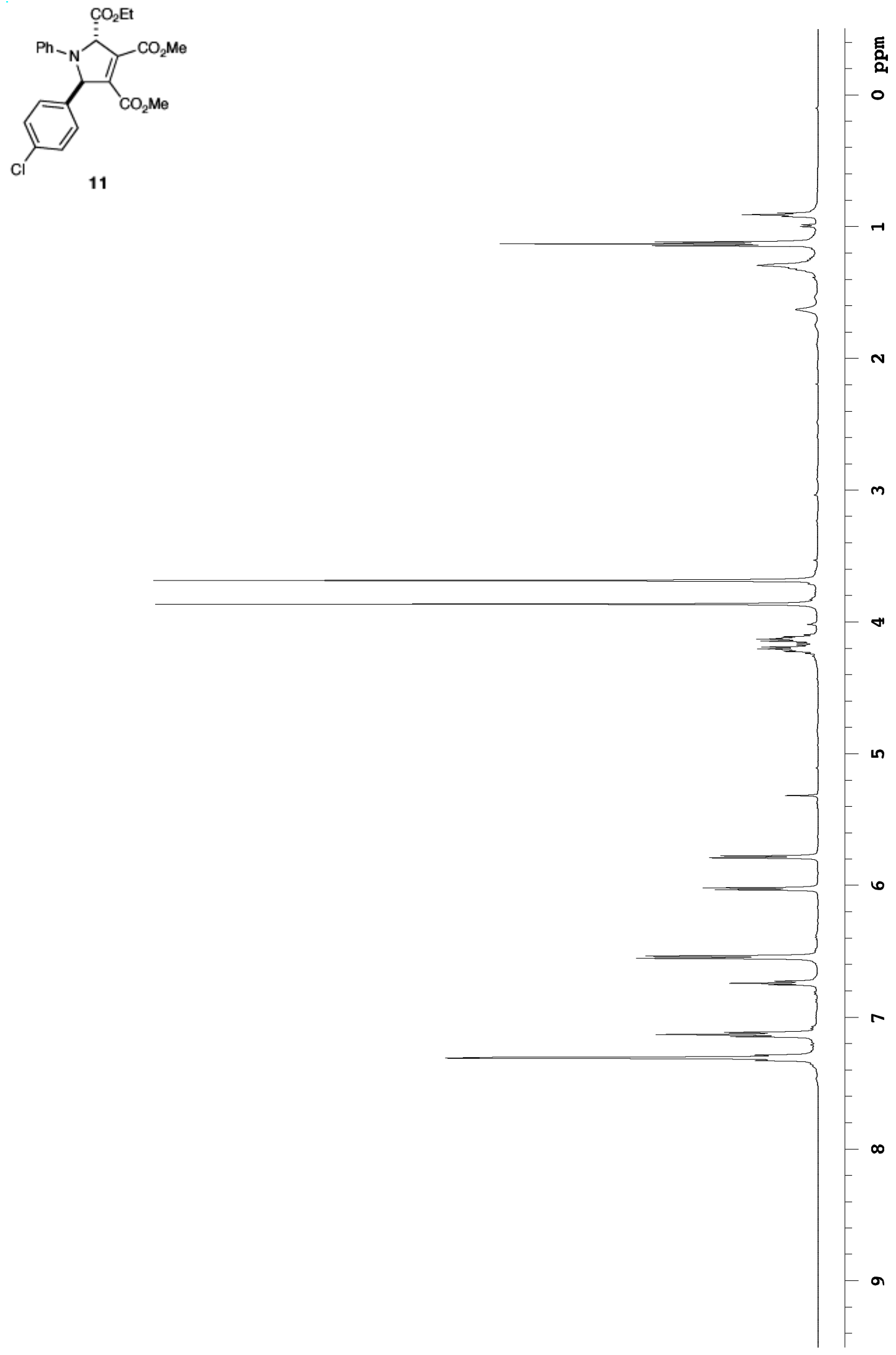


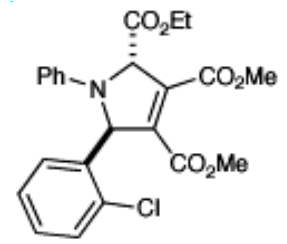

12
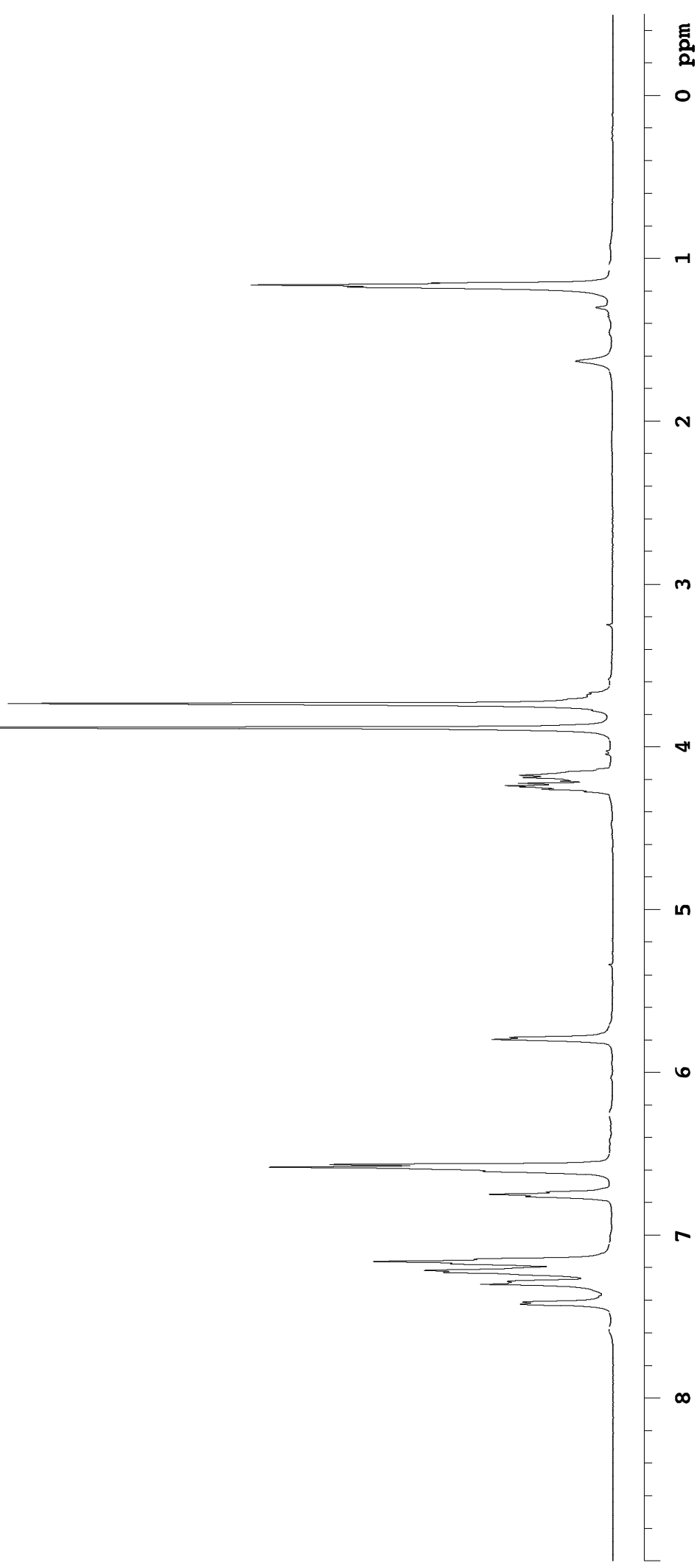


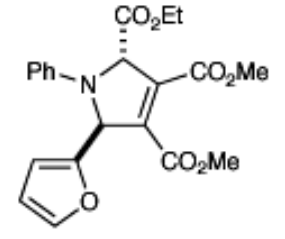

13
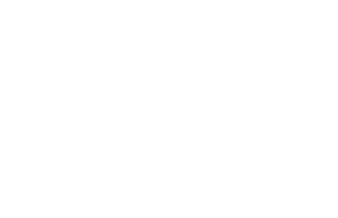
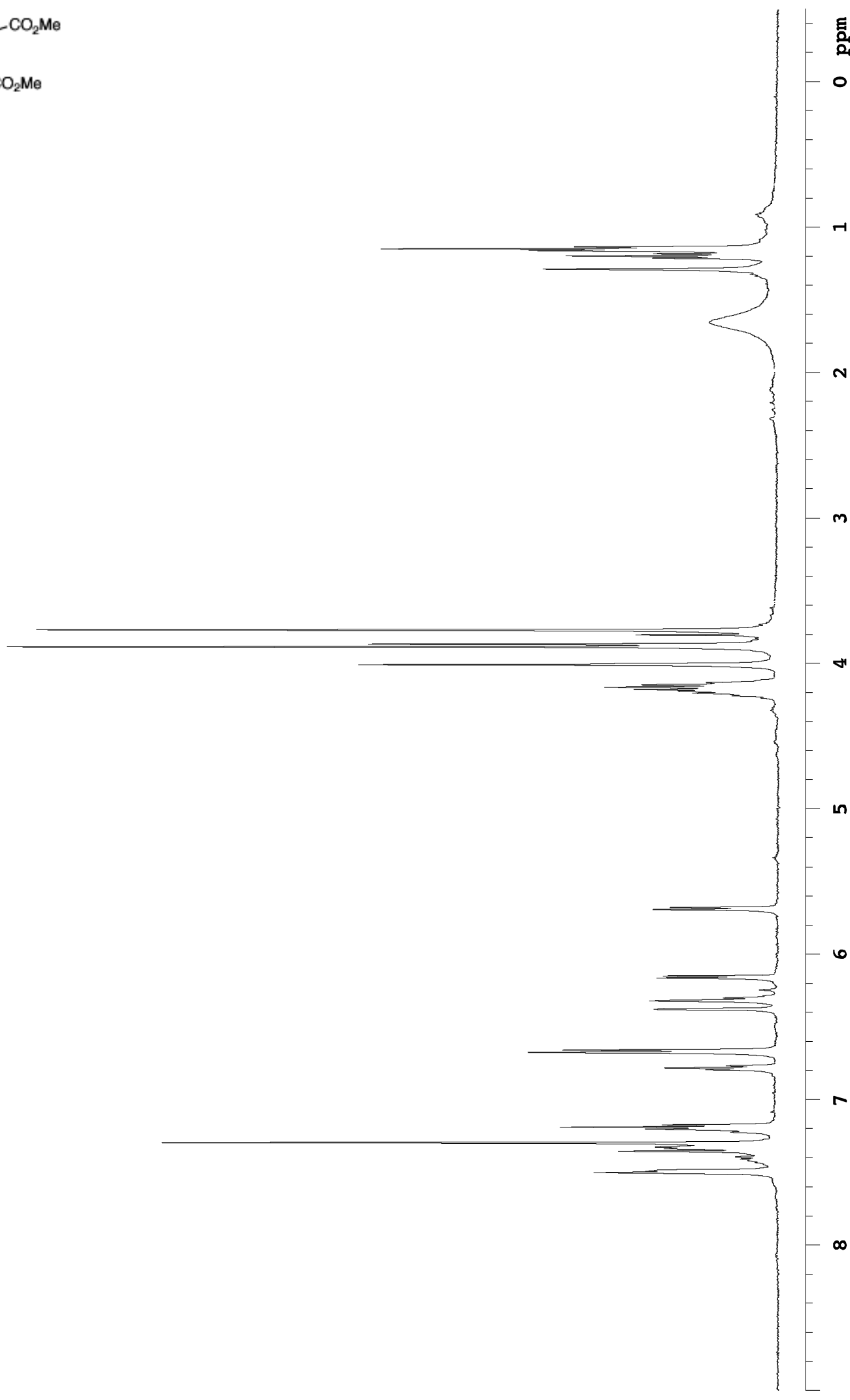

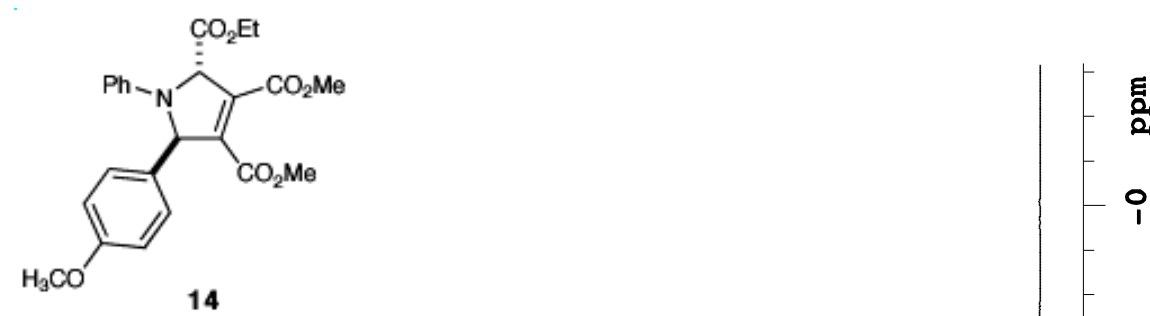

I

N

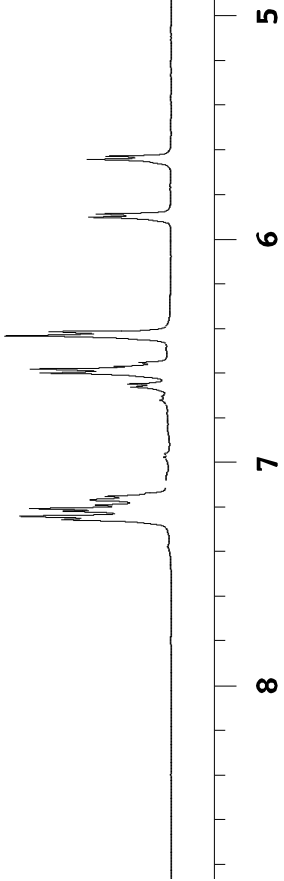




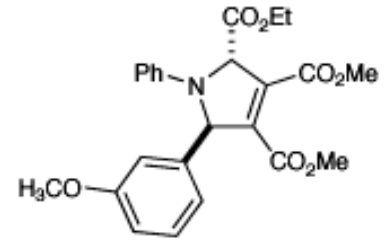

15

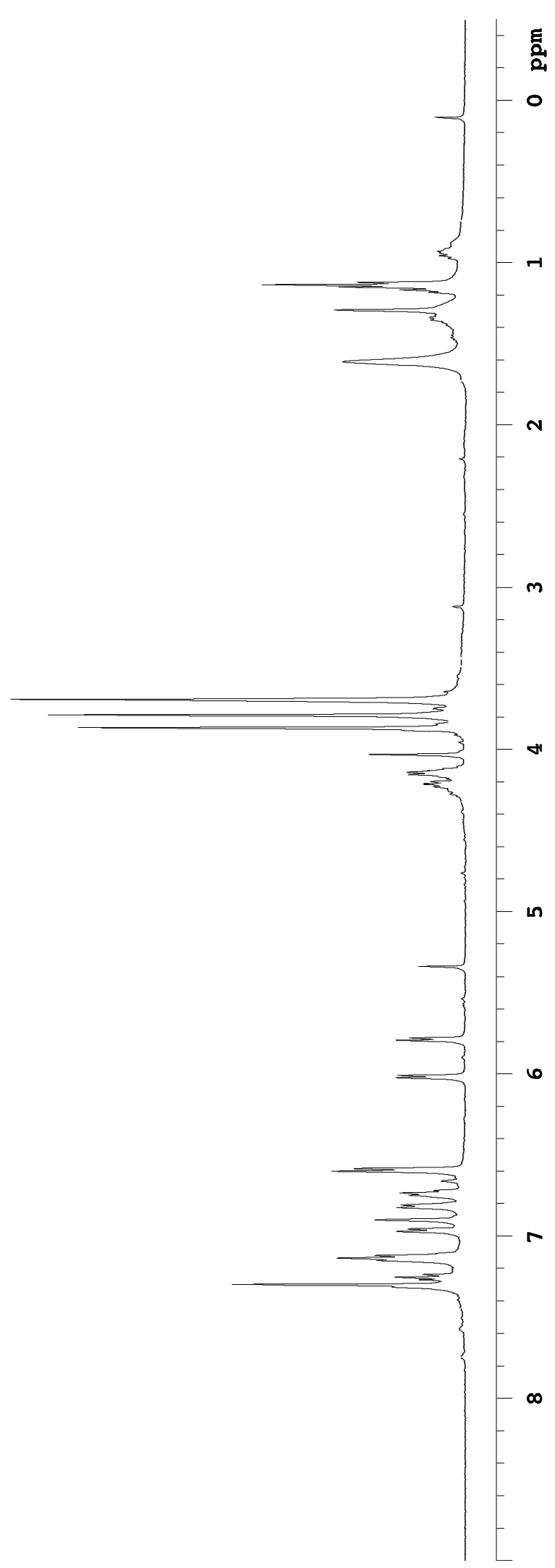




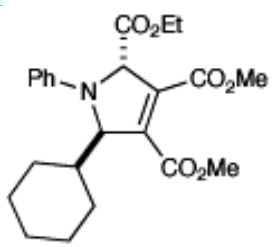

16

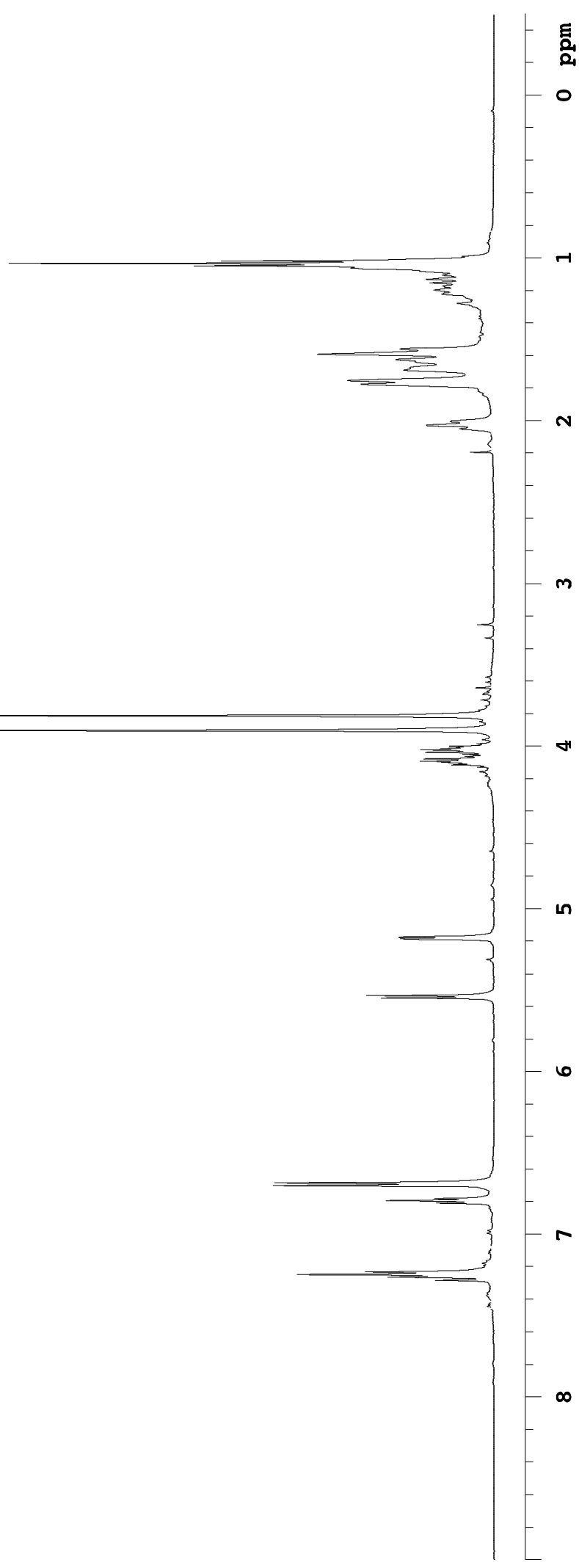



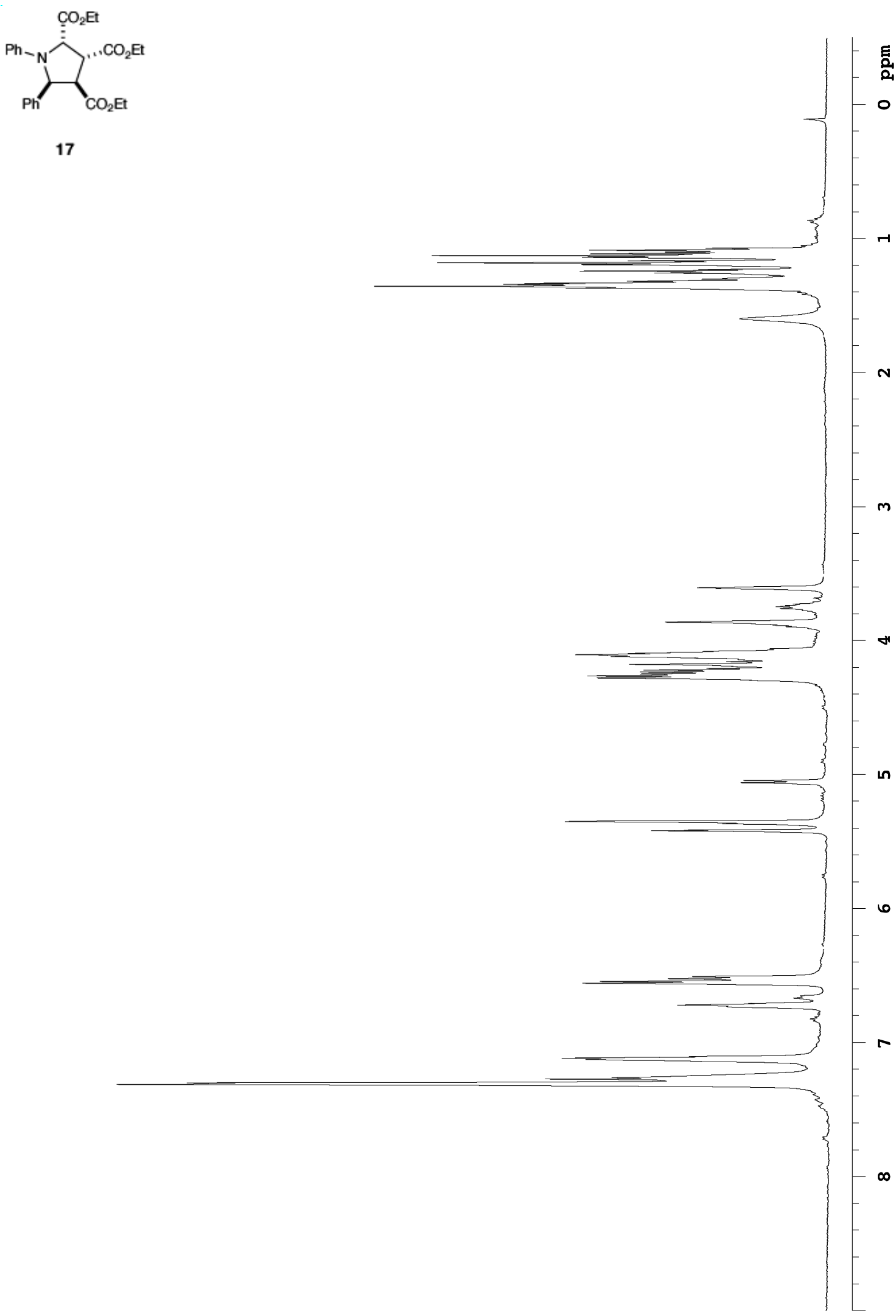


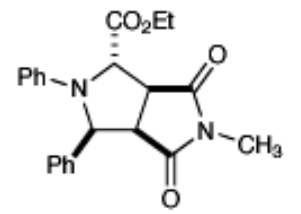

20

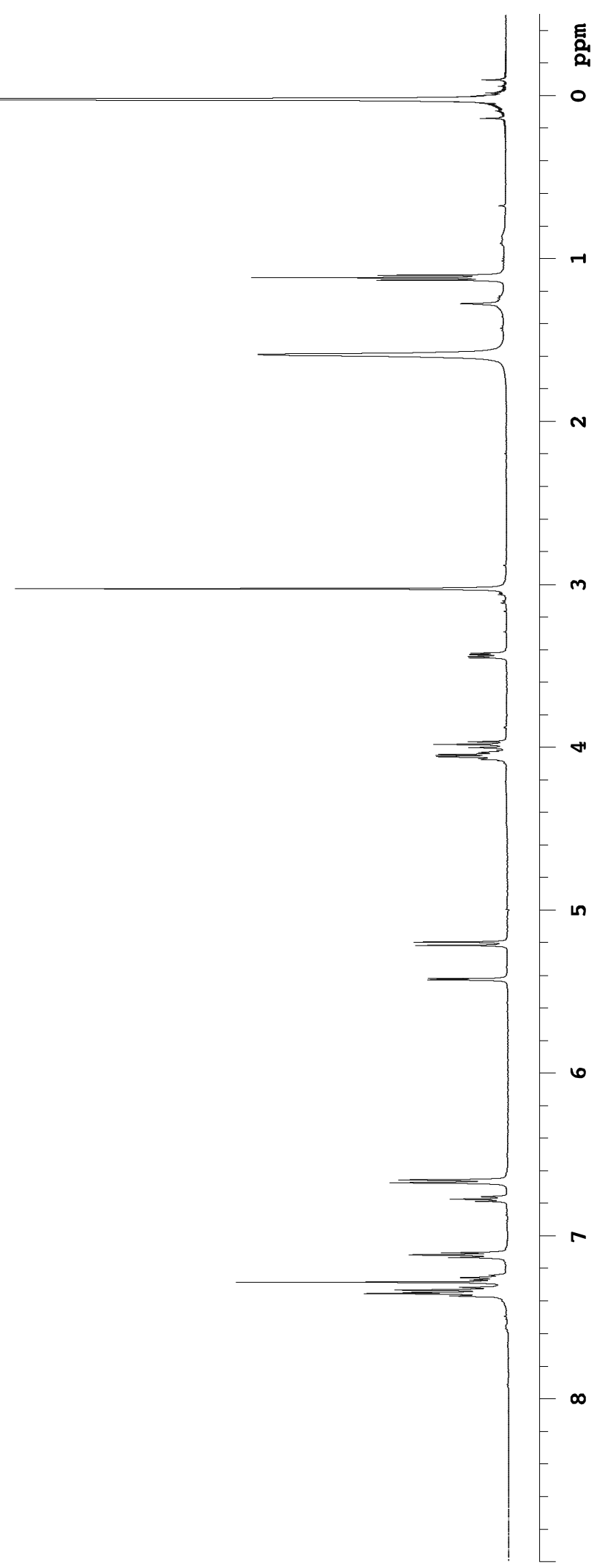



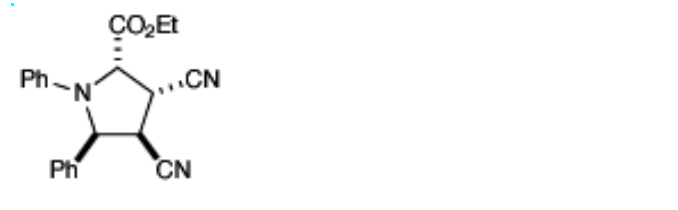

ถี

19

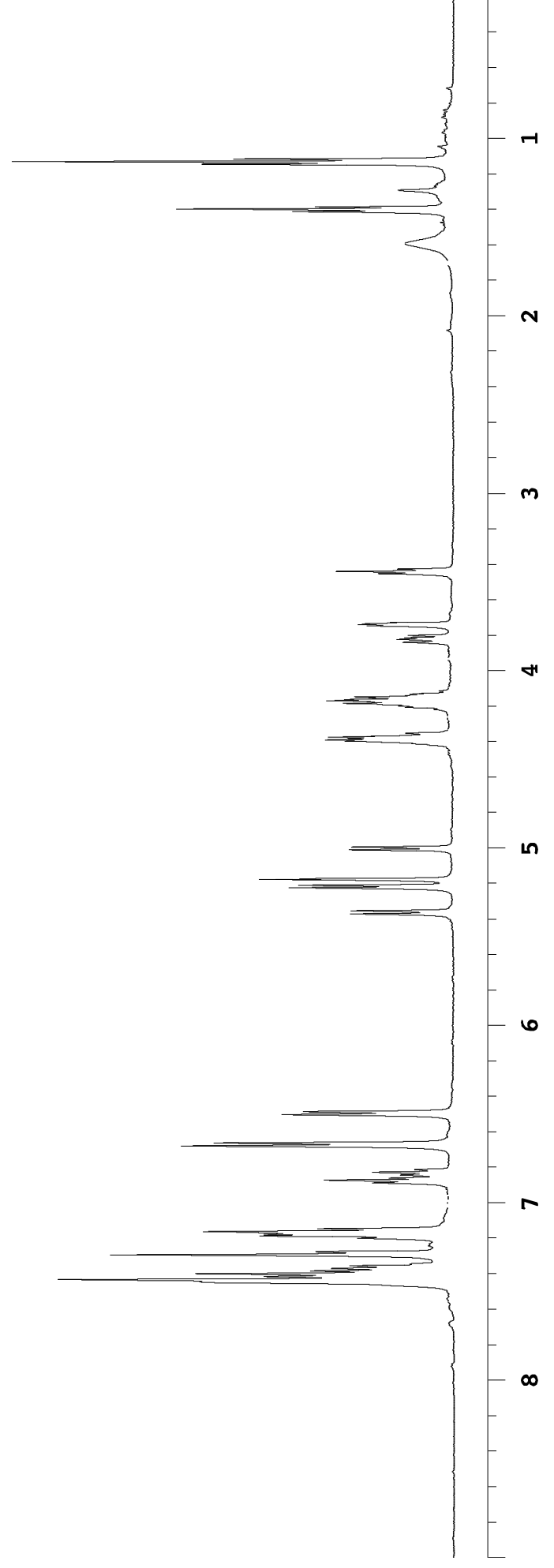


Relative Stereochemical assignment based on NMR Data comparison of pyrrolidines 23 and 25 to known compounds 25 and 26

The relative stereochemistry of pyrrolidine 23 was assigned by comparision $\left(500 \mathrm{MHz}{ }^{1} \mathrm{H} \mathrm{NMR}\right)$ to known pyrrolidine 24. Pyrrolidine 24 stereochemistry was assigned by Che and coworkers by comparison to the X-ray structure of the $N$-4-methoxyphenyl analog. ${ }^{5}$

\begin{tabular}{|l|l|l|l|}
\hline Scheidt & \multicolumn{1}{|c|}{${ }^{\mathrm{Ph}}$} & Che & \\
& & & \\
\hline 5.27 & $\mathrm{~d}, J=9.1 \mathrm{~Hz}, 1 \mathrm{H}$ & 5.28 & $\mathrm{~d}, J=9.0 \mathrm{~Hz}, 1 \mathrm{H}$ \\
\hline 4.73 & $\mathrm{~d}, J=8.6 \mathrm{~Hz}, 1 \mathrm{H}$ & 4.73 & $\mathrm{~d}, J=9.0 \mathrm{~Hz}, 1 \mathrm{H}$ \\
\hline 3.85 & $\mathrm{~m}, 1 \mathrm{H}$ & $3.91-3.84$ & $\mathrm{~m}, 1 \mathrm{H}$ \\
\hline $2.95-2.86$ & $\mathrm{ddd}, J=13.4,13.4,9.2 \mathrm{~Hz}, 1 \mathrm{H}$ & $2.96-2.84$ & $\mathrm{dt}, J=17.4,13.5 \mathrm{~Hz}, 1 \mathrm{H}$ \\
\hline 2.17 & $\mathrm{dd}, 12.8,6.1$ & 2.18 & $\mathrm{dd}, J=13.2 \mathrm{~Hz}, 6.3 \mathrm{~Hz}, 1 \mathrm{H}$ \\
\hline
\end{tabular}

Pyrrolidine 25 was synthesized by our standard catalytic conditions from $N$-benzylidene anisidine, EDA (2a), and $N$-phenylmaleamide, and then compared (500 $\mathrm{MHz}{ }^{1} \mathrm{H}$ NMR) to known pyrrolidine 26:

\begin{tabular}{|l|l|l|l|}
\hline Scheidt & & & \\
& & & \\
& & & \\
& $\mathrm{d}, J=4.5 \mathrm{~Hz}, 1 \mathrm{H}$ & 5.43 & $\mathrm{~d}, J=4.5 \mathrm{~Hz}, 1 \mathrm{H}$ \\
\hline 5.43 & $\mathrm{~d}, J=9.3 \mathrm{~Hz}, 1 \mathrm{H}$ & 5.19 & $\mathrm{~d}, J=9.3 \mathrm{~Hz}, 1 \mathrm{H}$ \\
\hline 5.20 & $\mathrm{dd}, J=9.3,9.3 \mathrm{~Hz}, 1 \mathrm{H}$ & $4.11-4.04$ & $\mathrm{~m}, 1 \mathrm{H}$ \\
\hline $4.13-4.18$ & $\mathrm{~s}, 3 \mathrm{H}$ & 3.68 & $\mathrm{~s}, 3 \mathrm{H}$ \\
\hline 3.77 & $\mathrm{~m}, 2 \mathrm{H}$ & $3.60-3.56$ & $\mathrm{~m}, 4 \mathrm{H}$ \\
\hline 3.47 & &
\end{tabular}

X-ray Crystallographic Data for Substituted Pyrrolidine 10:

(5) Li, G.-Y.; Chen, J.; Yu, W.-Y.; Hong, W.; Che, C.-M. Org. Lett. 2003, 5, 2153-2156. 


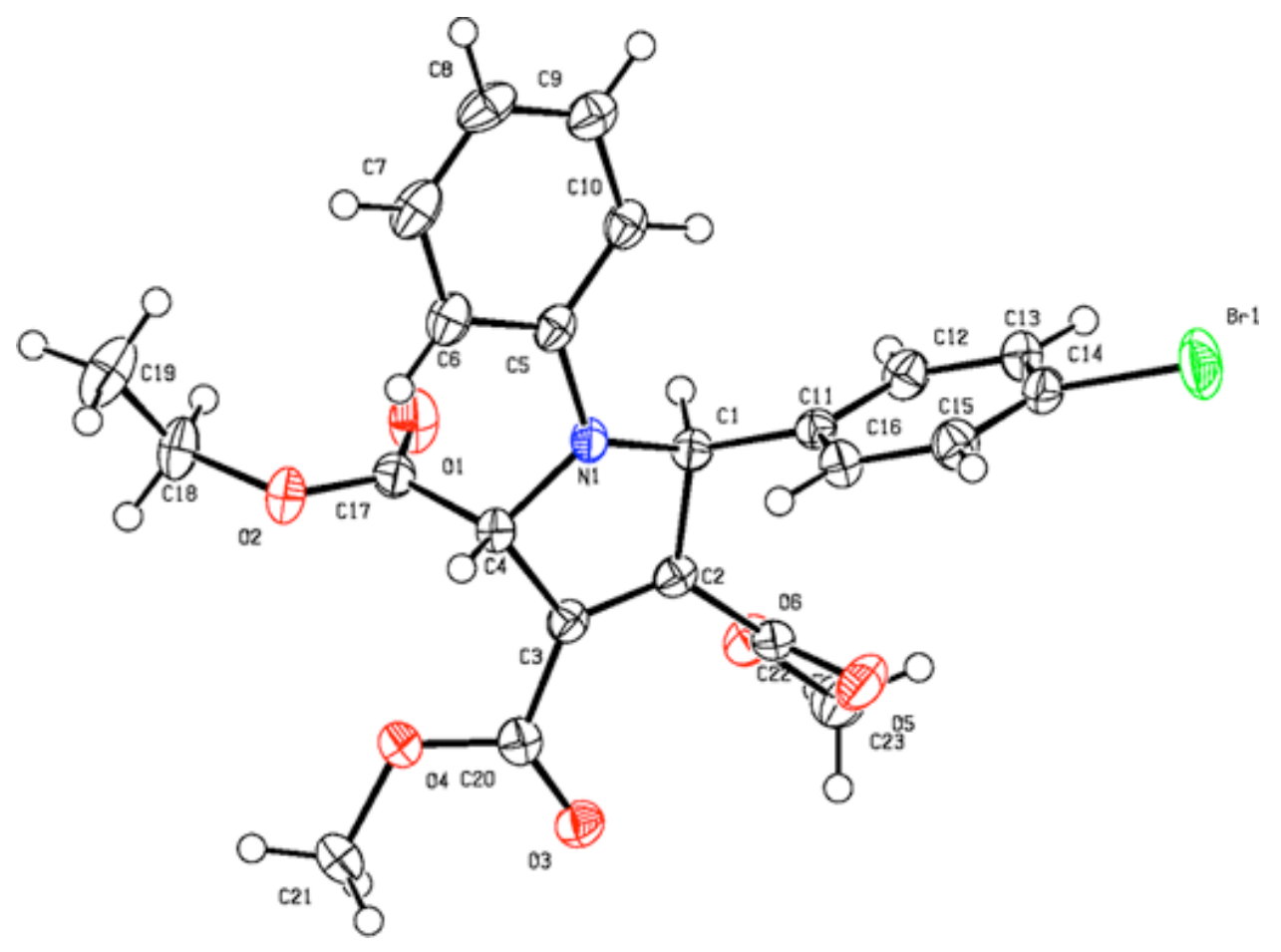

Crystal data and structure refinement for pyrrolidine $\mathbf{1 0}$.

Identification code $\quad \mathrm{s} 33 \mathrm{n} 1 \mathrm{~m}$

Empirical formula $\quad \mathrm{C} 23 \mathrm{H} 22 \mathrm{Br}$ N O6

Formula weight $\quad 488.33$

Temperature 153(2) K

Wavelength $\quad 0.71073 \AA$

Crystal system, space group Monoclinic, P2(1)/c

Unit cell dimensions

$$
\begin{aligned}
& \mathrm{a}=10.1952(17) \AA \\
& \mathrm{b}=12.015(2) \AA \quad=95.28(2)^{\mathrm{o}} \\
& \mathrm{c}=17.932(4) \AA
\end{aligned}
$$

Volume

$$
\text { 2187.2(8) А̊3 }
$$

Z, Calculated density

4, $1.483 \mathrm{Mg} / \mathrm{m} 3$

Absorption coefficient

$1.919 \mathrm{~mm}-1$

$\mathrm{F}(000)$

1000

Crystal size

$0.240 \times 0.104 \times 0.090 \mathrm{~mm}$

Theta range for data collection 2.01 to $28.85^{\circ}$ 
Limiting indices $\quad-13<=\mathrm{h}<=13,-15<=\mathrm{k}<=15,-23<=\mathrm{l}<=23$

Reflections collected / unique $19796 / 5306[\mathrm{R}(\mathrm{int})=0.0600]$

Completeness to theta $=28.85 \quad 92.5 \%$

Absorption correction Integration

Max. and min. transmission $\quad 0.8521$ and 0.7049

Refinement method Full-matrix least-squares on F2

Data / restraints / parameters 5306 / 0 / 283

Goodness-of-fit on $\mathrm{F}^{\wedge} 2 \quad 0.993$

Final $\mathrm{R}$ indices $[\mathrm{I}>2 \operatorname{sigma}(\mathrm{I})] \quad \mathrm{R} 1=0.0358, \mathrm{wR} 2=0.0785$

$\mathrm{R}$ indices (all data) $\quad \mathrm{R} 1=0.0770, \mathrm{wR}^{2}=0.0925$

Largest diff. peak and hole $\quad 0.520$ and -0.474 e- $/ \AA$-3

Table 2. Atomic coordinates and equivalent isotropic displacement parameters $\left(\mathrm{A}^{\wedge} 2 \times 10^{\wedge} 3\right)$ for $\mathbf{1 0}$.

$\mathrm{U}(\mathrm{eq})$ is defined as one third of the trace of the orthogonalized

Ui $J$ tensor.

$\begin{array}{llll}\mathrm{x} & \mathrm{y} & \mathrm{z} & \mathrm{U}\end{array}$

$\begin{array}{lllll}\mathrm{Br}(1) & 0.65539(3) & 1.03663(2) & 1.151831(16) & 49(1) \\ \mathrm{O}(1) & 0.66429(15) & 0.35665(13) & 0.88697(9) & 37(1) \\ \mathrm{O}(2) & 0.86117(14) & 0.27323(11) & 0.91350(8) & 29(1) \\ \mathrm{O}(3) & 0.92955(15) & 0.62024(12) & 0.76520(8) & 29(1) \\ \mathrm{O}(4) & 0.96641(15) & 0.44350(12) & 0.80431(9) & 31(1) \\ \mathrm{O}(5) & 0.76236(17) & 0.82423(12) & 0.85729(9) & 38(1) \\ \mathrm{O}(6) & 0.63890(14) & 0.70728(12) & 0.78170(8) & 29(1) \\ \mathrm{N}(1) & 0.78066(17) & 0.52306(13) & 0.99431(9) & 21(1) \\ \mathrm{C}(1) & 0.6954(2) & 0.61169(16) & 0.96109(11) & 21(1) \\ \mathrm{C}(2) & 0.7550(2) & 0.63223(16) & 0.88855(11) & 21(1)\end{array}$




$\begin{array}{lllll}\mathrm{C}(3) & 0.8410(2) & 0.55321(16) & 0.87513(12) & 22(1) \\ \mathrm{C}(4) & 0.8497(2) & 0.46714(16) & 0.93645(11) & 22(1) \\ \mathrm{C}(5) & 0.7457(2) & 0.46219(16) & 1.05640(11) & 22(1) \\ \mathrm{C}(6) & 0.8216(2) & 0.37050(17) & 1.08226(12) & 28(1) \\ \mathrm{C}(7) & 0.7899(2) & 0.31197(18) & 1.14460(13) & 33(1) \\ \mathrm{C}(8) & 0.6834(2) & 0.34226(19) & 1.18240(13) & 34(1) \\ \mathrm{C}(9) & 0.6086(2) & 0.43306(18) & 1.15730(12) & 30(1) \\ \mathrm{C}(10) & 0.6384(2) & 0.49271(17) & 1.09499(12) & 25(1) \\ \mathrm{C}(11) & 0.6883(2) & 0.71518(16) & 1.00939(11) & 21(1) \\ \mathrm{C}(12) & 0.5711(2) & 0.77462(17) & 1.00541(12) & 28(1) \\ \mathrm{C}(13) & 0.5614(2) & 0.87145(17) & 1.04757(13) & 31(1) \\ \mathrm{C}(14) & 0.6690(2) & 0.90539(17) & 1.09369(13) & 29(1) \\ \mathrm{C}(15) & 0.7865(2) & 0.84773(18) & 1.09839(12) & 30(1) \\ \mathrm{C}(16) & 0.7954(2) & 0.75196(17) & 1.05548(12) & 26(1) \\ \mathrm{C}(17) & 0.7787(2) & 0.35945(17) & 0.90870(11) & 23(1) \\ \mathrm{C}(18) & 0.8100(3) & 0.16400(17) & 0.88985(14) & 37(1) \\ \mathrm{C}(19) & 0.8402(3) & 0.0838(2) & 0.95265(16) & 53(1) \\ \mathrm{C}(20) & 0.9159(2) & 0.54553(17) & 0.80870(12) & 24(1) \\ \mathrm{C}(21) & 1.0472(2) & 0.4234(2) & 0.74318(14) & 37(1) \\ \mathrm{C}(22) & 0.7210(2) & 0.73289(17) & 0.84101(12) & 25(1) \\ \mathrm{C}(23) & 0.6012(2) & 0.79958(19) & 0.73200(13) & 37(1)\end{array}$

Table 3. Bond lengths [A] and angles [deg] for $\mathbf{1 0 .}$

\begin{tabular}{ll}
\hline $\mathrm{Br}(1)-\mathrm{C}(14)$ & $1.902(2)$ \\
$\mathrm{O}(1)-\mathrm{C}(17)$ & $1.196(2)$ \\
$\mathrm{O}(2)-\mathrm{C}(17)$ & $1.332(2)$ \\
$\mathrm{O}(2)-\mathrm{C}(18)$ & $1.461(2)$ \\
$\mathrm{O}(3)-\mathrm{C}(20)$ & $1.206(2)$ \\
$\mathrm{O}(4)-\mathrm{C}(20)$ & $1.335(2)$
\end{tabular}




\begin{tabular}{|c|c|}
\hline $\mathrm{O}(4)-\mathrm{C}(21)$ & $1.451(3)$ \\
\hline $\mathrm{O}(5)-\mathrm{C}(22)$ & $1.202(2)$ \\
\hline $\mathrm{O}(6)-\mathrm{C}(22)$ & $1.327(3)$ \\
\hline $\mathrm{O}(6)-\mathrm{C}(23)$ & $1.452(2)$ \\
\hline $\mathrm{N}(1)-\mathrm{C}(5)$ & $1.405(3)$ \\
\hline $\mathrm{N}(1)-\mathrm{C}(1)$ & $1.466(2)$ \\
\hline $\mathrm{N}(1)-\mathrm{C}(4)$ & $1.469(3)$ \\
\hline$C(1)-C(2)$ & $1.506(3)$ \\
\hline $\mathrm{C}(1)-\mathrm{C}(11)$ & $1.521(3)$ \\
\hline $\mathrm{C}(1)-\mathrm{H}(1)$ & 1.0000 \\
\hline$C(2)-C(3)$ & $1.330(3)$ \\
\hline $\mathrm{C}(2)-\mathrm{C}(22)$ & $1.502(3)$ \\
\hline$C(3)-C(20)$ & $1.476(3)$ \\
\hline$C(3)-C(4)$ & $1.506(3)$ \\
\hline $\mathrm{C}(4)-\mathrm{C}(17)$ & $1.543(3)$ \\
\hline $\mathrm{C}(4)-\mathrm{H}(4)$ & 1.0000 \\
\hline $\mathrm{C}(5)-\mathrm{C}(10)$ & $1.397(3)$ \\
\hline$C(5)-C(6)$ & $1.401(3)$ \\
\hline$C(6)-C(7)$ & $1.384(3)$ \\
\hline $\mathrm{C}(6)-\mathrm{H}(6)$ & 0.9500 \\
\hline$C(7)-C(8)$ & $1.381(3)$ \\
\hline $\mathrm{C}(7)-\mathrm{H}(7)$ & 0.9500 \\
\hline$C(8)-C(9)$ & $1.382(3)$ \\
\hline $\mathrm{C}(8)-\mathrm{H}(8)$ & 0.9500 \\
\hline $\mathrm{C}(9)-\mathrm{C}(10)$ & $1.384(3)$ \\
\hline $\mathrm{C}(9)-\mathrm{H}(9)$ & 0.9500 \\
\hline $\mathrm{C}(10)-\mathrm{H}(10)$ & 0.9500 \\
\hline $\mathrm{C}(11)-\mathrm{C}(16)$ & $1.380(3)$ \\
\hline$C(11)-C(12)$ & $1.388(3)$ \\
\hline $\mathrm{C}(12)-\mathrm{C}(13)$ & $1.396(3)$ \\
\hline
\end{tabular}




\begin{tabular}{|c|c|}
\hline $\mathrm{C}(12)-\mathrm{H}(12)$ & 0.9500 \\
\hline$C(13)-C(14)$ & $1.374(3)$ \\
\hline $\mathrm{C}(13)-\mathrm{H}(13)$ & 0.9500 \\
\hline$C(14)-C(15)$ & $1.380(3)$ \\
\hline$C(15)-C(16)$ & $1.392(3)$ \\
\hline $\mathrm{C}(15)-\mathrm{H}(15)$ & 0.9500 \\
\hline $\mathrm{C}(16)-\mathrm{H}(16)$ & 0.9500 \\
\hline $\mathrm{C}(18)-\mathrm{C}(19)$ & $1.492(3)$ \\
\hline $\mathrm{C}(18)-\mathrm{H}(18 \mathrm{~A})$ & 0.9900 \\
\hline $\mathrm{C}(18)-\mathrm{H}(18 \mathrm{~B})$ & 0.9900 \\
\hline $\mathrm{C}(19)-\mathrm{H}(19 \mathrm{~A})$ & 0.9800 \\
\hline $\mathrm{C}(19)-\mathrm{H}(19 \mathrm{~B})$ & 0.9800 \\
\hline $\mathrm{C}(19)-\mathrm{H}(19 \mathrm{C})$ & 0.9800 \\
\hline $\mathrm{C}(21)-\mathrm{H}(21 \mathrm{~A})$ & 0.9800 \\
\hline $\mathrm{C}(21)-\mathrm{H}(21 \mathrm{~B})$ & 0.9800 \\
\hline $\mathrm{C}(21)-\mathrm{H}(21 \mathrm{C})$ & 0.9800 \\
\hline $\mathrm{C}(23)-\mathrm{H}(23 \mathrm{~A})$ & 0.9800 \\
\hline $\mathrm{C}(23)-\mathrm{H}(23 \mathrm{~B})$ & 0.9800 \\
\hline $\mathrm{C}(23)-\mathrm{H}(23 \mathrm{C})$ & 0.9800 \\
\hline $\mathrm{C}(17)-\mathrm{O}(2)-\mathrm{C}(18)$ & $118.24(16)$ \\
\hline $\mathrm{C}(20)-\mathrm{O}(4)-\mathrm{C}(21)$ & $116.51(17)$ \\
\hline $\mathrm{C}(22)-\mathrm{O}(6)-\mathrm{C}(23)$ & $115.24(17)$ \\
\hline $\mathrm{C}(5)-\mathrm{N}(1)-\mathrm{C}(1)$ & $121.06(16)$ \\
\hline $\mathrm{C}(5)-\mathrm{N}(1)-\mathrm{C}(4)$ & 119.64(16) \\
\hline $\mathrm{C}(1)-\mathrm{N}(1)-\mathrm{C}(4)$ & $110.49(15)$ \\
\hline $\mathrm{N}(1)-\mathrm{C}(1)-\mathrm{C}(2)$ & $101.47(16)$ \\
\hline $\mathrm{N}(1)-\mathrm{C}(1)-\mathrm{C}(11)$ & $114.84(16)$ \\
\hline $\mathrm{C}(2)-\mathrm{C}(1)-\mathrm{C}(11)$ & 113.71(16) \\
\hline $\mathrm{N}(1)-\mathrm{C}(1)-\mathrm{H}(1)$ & 108.8 \\
\hline $\mathrm{C}(2)-\mathrm{C}(1)-\mathrm{H}(1)$ & 108.8 \\
\hline
\end{tabular}




\begin{tabular}{|c|c|}
\hline $\mathrm{C}(11)-\mathrm{C}(1)-\mathrm{H}(1)$ & 108.8 \\
\hline $\mathrm{C}(3)-\mathrm{C}(2)-\mathrm{C}(22)$ & $126.43(19)$ \\
\hline $\mathrm{C}(3)-\mathrm{C}(2)-\mathrm{C}(1)$ & 111.39(18) \\
\hline $\mathrm{C}(22)-\mathrm{C}(2)-\mathrm{C}(1)$ & $122.14(17)$ \\
\hline$C(2)-C(3)-C(20)$ & $126.15(19)$ \\
\hline$C(2)-C(3)-C(4)$ & $110.70(19)$ \\
\hline $\mathrm{C}(20)-\mathrm{C}(3)-\mathrm{C}(4)$ & $123.12(18)$ \\
\hline $\mathrm{N}(1)-\mathrm{C}(4)-\mathrm{C}(3)$ & $101.65(15)$ \\
\hline $\mathrm{N}(1)-\mathrm{C}(4)-\mathrm{C}(17)$ & $111.74(16)$ \\
\hline$C(3)-C(4)-C(17)$ & $110.24(17)$ \\
\hline $\mathrm{N}(1)-\mathrm{C}(4)-\mathrm{H}(4)$ & 111.0 \\
\hline $\mathrm{C}(3)-\mathrm{C}(4)-\mathrm{H}(4)$ & 111.0 \\
\hline $\mathrm{C}(17)-\mathrm{C}(4)-\mathrm{H}(4)$ & 111.0 \\
\hline$C(10)-C(5)-C(6)$ & $118.47(19)$ \\
\hline $\mathrm{C}(10)-\mathrm{C}(5)-\mathrm{N}(1)$ & $121.56(18)$ \\
\hline$C(6)-C(5)-N(1)$ & 119.94(19) \\
\hline$C(7)-C(6)-C(5)$ & $120.3(2)$ \\
\hline$C(7)-C(6)-H(6)$ & 119.9 \\
\hline $\mathrm{C}(5)-\mathrm{C}(6)-\mathrm{H}(6)$ & 119.9 \\
\hline$C(8)-C(7)-C(6)$ & $121.0(2)$ \\
\hline $\mathrm{C}(8)-\mathrm{C}(7)-\mathrm{H}(7)$ & 119.5 \\
\hline $\mathrm{C}(6)-\mathrm{C}(7)-\mathrm{H}(7)$ & 119.5 \\
\hline$C(7)-C(8)-C(9)$ & $118.9(2)$ \\
\hline $\mathrm{C}(7)-\mathrm{C}(8)-\mathrm{H}(8)$ & 120.5 \\
\hline $\mathrm{C}(9)-\mathrm{C}(8)-\mathrm{H}(8)$ & 120.5 \\
\hline $\mathrm{C}(8)-\mathrm{C}(9)-\mathrm{C}(10)$ & $121.1(2)$ \\
\hline $\mathrm{C}(8)-\mathrm{C}(9)-\mathrm{H}(9)$ & 119.5 \\
\hline $\mathrm{C}(10)-\mathrm{C}(9)-\mathrm{H}(9)$ & 119.5 \\
\hline$C(9)-C(10)-C(5)$ & $120.3(2)$ \\
\hline $\mathrm{C}(9)-\mathrm{C}(10)-\mathrm{H}(10)$ & 119.9 \\
\hline
\end{tabular}




\begin{tabular}{|c|c|}
\hline $\mathrm{C}(5)-\mathrm{C}(10)-\mathrm{H}(10)$ & 119.9 \\
\hline$C(16)-C(11)-C(12)$ & $119.74(19)$ \\
\hline$C(16)-C(11)-C(1)$ & $121.74(18)$ \\
\hline $\mathrm{C}(12)-\mathrm{C}(11)-\mathrm{C}(1)$ & $118.52(18)$ \\
\hline$C(11)-C(12)-C(13)$ & $120.3(2)$ \\
\hline $\mathrm{C}(11)-\mathrm{C}(12)-\mathrm{H}(12)$ & 119.9 \\
\hline $\mathrm{C}(13)-\mathrm{C}(12)-\mathrm{H}(12)$ & 119.9 \\
\hline$C(14)-C(13)-C(12)$ & $118.7(2)$ \\
\hline $\mathrm{C}(14)-\mathrm{C}(13)-\mathrm{H}(13)$ & 120.6 \\
\hline $\mathrm{C}(12)-\mathrm{C}(13)-\mathrm{H}(13)$ & 120.6 \\
\hline $\mathrm{C}(13)-\mathrm{C}(14)-\mathrm{C}(15)$ & $121.9(2)$ \\
\hline $\mathrm{C}(13)-\mathrm{C}(14)-\mathrm{Br}(1)$ & $118.76(17)$ \\
\hline$C(15)-C(14)-B r(1)$ & $119.31(17)$ \\
\hline$C(14)-C(15)-C(16)$ & $118.8(2)$ \\
\hline $\mathrm{C}(14)-\mathrm{C}(15)-\mathrm{H}(15)$ & 120.6 \\
\hline $\mathrm{C}(16)-\mathrm{C}(15)-\mathrm{H}(15)$ & 120.6 \\
\hline$C(11)-C(16)-C(15)$ & $120.5(2)$ \\
\hline $\mathrm{C}(11)-\mathrm{C}(16)-\mathrm{H}(16)$ & 119.7 \\
\hline $\mathrm{C}(15)-\mathrm{C}(16)-\mathrm{H}(16)$ & 119.7 \\
\hline $\mathrm{O}(1)-\mathrm{C}(17)-\mathrm{O}(2)$ & $126.2(2)$ \\
\hline $\mathrm{O}(1)-\mathrm{C}(17)-\mathrm{C}(4)$ & $122.93(19)$ \\
\hline $\mathrm{O}(2)-\mathrm{C}(17)-\mathrm{C}(4)$ & $110.88(17)$ \\
\hline $\mathrm{O}(2)-\mathrm{C}(18)-\mathrm{C}(19)$ & $108.50(19)$ \\
\hline $\mathrm{O}(2)-\mathrm{C}(18)-\mathrm{H}(18 \mathrm{~A})$ & 110.0 \\
\hline $\mathrm{C}(19)-\mathrm{C}(18)-\mathrm{H}(18 \mathrm{~A})$ & 110.0 \\
\hline $\mathrm{O}(2)-\mathrm{C}(18)-\mathrm{H}(18 \mathrm{~B})$ & 110.0 \\
\hline $\mathrm{C}(19)-\mathrm{C}(18)-\mathrm{H}(18 \mathrm{~B})$ & 110.0 \\
\hline $\mathrm{H}(18 \mathrm{~A})-\mathrm{C}(18)-\mathrm{H}(18 \mathrm{~B})$ & 108.4 \\
\hline $\mathrm{C}(18)-\mathrm{C}(19)-\mathrm{H}(19 \mathrm{~A})$ & 109.5 \\
\hline $\mathrm{C}(18)-\mathrm{C}(19)-\mathrm{H}(19 \mathrm{~B})$ & 109.5 \\
\hline
\end{tabular}




\begin{tabular}{|c|c|}
\hline $\mathrm{H}(19 \mathrm{~A})-\mathrm{C}(19)-\mathrm{H}(19 \mathrm{~B})$ & 109.5 \\
\hline $\mathrm{C}(18)-\mathrm{C}(19)-\mathrm{H}(19 \mathrm{C})$ & 109.5 \\
\hline $\mathrm{H}(19 \mathrm{~A})-\mathrm{C}(19)-\mathrm{H}(19 \mathrm{C})$ & 109.5 \\
\hline $\mathrm{H}(19 \mathrm{~B})-\mathrm{C}(19)-\mathrm{H}(19 \mathrm{C})$ & 109.5 \\
\hline $\mathrm{O}(3)-\mathrm{C}(20)-\mathrm{O}(4)$ & $125.3(2)$ \\
\hline $\mathrm{O}(3)-\mathrm{C}(20)-\mathrm{C}(3)$ & $125.08(19)$ \\
\hline $\mathrm{O}(4)-\mathrm{C}(20)-\mathrm{C}(3)$ & $109.63(18)$ \\
\hline $\mathrm{O}(4)-\mathrm{C}(21)-\mathrm{H}(21 \mathrm{~A})$ & 109.5 \\
\hline $\mathrm{O}(4)-\mathrm{C}(21)-\mathrm{H}(21 \mathrm{~B})$ & 109.5 \\
\hline $\mathrm{H}(21 \mathrm{~A})-\mathrm{C}(21)-\mathrm{H}(21 \mathrm{~B})$ & 109.5 \\
\hline $\mathrm{O}(4)-\mathrm{C}(21)-\mathrm{H}(21 \mathrm{C})$ & 109.5 \\
\hline$H(21 A)-C(21)-H(21 C)$ & 109.5 \\
\hline $\mathrm{H}(21 \mathrm{~B})-\mathrm{C}(21)-\mathrm{H}(21 \mathrm{C})$ & 109.5 \\
\hline $\mathrm{O}(5)-\mathrm{C}(22)-\mathrm{O}(6)$ & $126.0(2)$ \\
\hline $\mathrm{O}(5)-\mathrm{C}(22)-\mathrm{C}(2)$ & $122.7(2)$ \\
\hline $\mathrm{O}(6)-\mathrm{C}(22)-\mathrm{C}(2)$ & $111.32(18)$ \\
\hline $\mathrm{O}(6)-\mathrm{C}(23)-\mathrm{H}(23 \mathrm{~A})$ & 109.5 \\
\hline $\mathrm{O}(6)-\mathrm{C}(23)-\mathrm{H}(23 \mathrm{~B})$ & 109.5 \\
\hline $\mathrm{H}(23 \mathrm{~A})-\mathrm{C}(23)-\mathrm{H}(23 \mathrm{~B})$ & 109.5 \\
\hline $\mathrm{O}(6)-\mathrm{C}(23)-\mathrm{H}(23 \mathrm{C})$ & 109.5 \\
\hline $\mathrm{H}(23 \mathrm{~A})-\mathrm{C}(23)-\mathrm{H}(23 \mathrm{C})$ & 109.5 \\
\hline $\mathrm{H}(23 \mathrm{~B})-\mathrm{C}(23)-\mathrm{H}(23 \mathrm{C})$ & 109.5 \\
\hline
\end{tabular}

Symmetry transformations used to generate equivalent atoms:

Table 4. Anisotropic displacement parameters $\left(\mathrm{A}^{\wedge} 2 \times 10^{\wedge} 3\right)$ for $\mathbf{1 0}$.

The anisotropic displacement factor exponent takes the form:

$-2 \mathrm{pi}^{\wedge} 2\left[\mathrm{~h}^{\wedge} 2 \mathrm{a}^{* \wedge} 2 \mathrm{U} 11+\ldots+2 \mathrm{~h} \mathrm{k} \mathrm{a} \mathrm{b}^{*} \mathrm{U} 12\right]$ 
$\begin{array}{llllll}\text { U11 } & \text { U22 } & \text { U33 } & \text { U23 } & \text { U13 } & \text { U12 }\end{array}$

$\begin{array}{lcccccc}\mathrm{Br}(1) & 61(1) & 30(1) & 57(1) & -18(1) & 9(1) & 6(1) \\ \mathrm{O}(1) & 26(1) & 31(1) & 53(1) & -9(1) & -8(1) & 0(1) \\ \mathrm{O}(2) & 32(1) & 19(1) & 34(1) & -3(1) & -3(1) & 2(1) \\ \mathrm{O}(3) & 35(1) & 27(1) & 26(1) & 4(1) & 5(1) & 0(1) \\ \mathrm{O}(4) & 36(1) & 25(1) & 33(1) & -1(1) & 13(1) & 3(1) \\ \mathrm{O}(5) & 61(1) & 24(1) & 29(1) & 2(1) & 6(1) & -8(1) \\ \mathrm{O}(6) & 31(1) & 29(1) & 27(1) & 9(1) & -3(1) & 3(1) \\ \mathrm{N}(1) & 28(1) & 17(1) & 18(1) & 0(1) & 2(1) & 3(1) \\ \mathrm{C}(1) & 23(1) & 21(1) & 18(1) & 1(1) & 0(1) & 1(1) \\ \mathrm{C}(2) & 23(1) & 21(1) & 19(1) & 0(1) & 0(1) & -4(1) \\ \mathrm{C}(3) & 25(1) & 20(1) & 20(1) & 0(1) & 1(1) & -1(1) \\ \mathrm{C}(4) & 23(1) & 20(1) & 23(1) & -2(1) & 1(1) & 1(1) \\ \mathrm{C}(5) & 28(1) & 19(1) & 19(1) & -1(1) & -3(1) & -4(1) \\ \mathrm{C}(6) & 31(1) & 23(1) & 28(1) & 1(1) & -5(1) & -1(1) \\ \mathrm{C}(7) & 39(1) & 26(1) & 32(1) & 7(1) & -11(1) & -4(1) \\ \mathrm{C}(8) & 45(2) & 33(1) & 22(1) & 6(1) & -4(1) & -14(1) \\ \mathrm{C}(9) & 38(1) & 28(1) & 23(1) & -3(1) & 1(1) & -10(1) \\ \mathrm{C}(10) & 30(1) & 21(1) & 23(1) & -2(1) & -2(1) & -3(1) \\ \mathrm{C}(11) & 26(1) & 20(1) & 17(1) & 2(1) & 3(1) & 0(1) \\ \mathrm{C}(12) & 27(1) & 26(1) & 31(1) & 1(1) & -1(1) & -1(1) \\ \mathrm{C}(13) & 32(1) & 23(1) & 40(1) & 3(1) & 8(1) & 5(1) \\ \mathrm{C}(14) & 39(1) & 20(1) & 29(1) & -3(1) & 9(1) & -1(1) \\ \mathrm{C}(15) & 34(1) & 28(1) & 27(1) & -5(1) & 1(1) & -3(1) \\ \mathrm{C}(16) & 25(1) & 24(1) & 27(1) & -2(1) & 2(1) & 2(1) \\ \mathrm{C}(17) & 29(1) & 22(1) & 19(1) & 0(1) & 2(1) & 1(1) \\ \mathrm{C}(18) & 46(2) & 19(1) & 44(2) & -7(1) & -1(1) & -2(1) \\ \mathrm{C}(19) & 72(2) & 27(1) & 57(2) & 10(1) & -5(2) & -8(1)\end{array}$




$\begin{array}{lllllll}\mathrm{C}(20) & 23(1) & 25(1) & 25(1) & -3(1) & -2(1) & -1(1) \\ \mathrm{C}(21) & 41(1) & 33(1) & 41(2) & -6(1) & 18(1) & 4(1) \\ \mathrm{C}(22) & 29(1) & 23(1) & 25(1) & 3(1) & 9(1) & 2(1) \\ \mathrm{C}(23) & 41(1) & 38(1) & 34(1) & 15(1) & 3(1) & 11(1)\end{array}$

Table 5. Hydrogen coordinates ( x 10^4) and isotropic displacement parameters $\left(\mathrm{A}^{\wedge} 2 \times 10^{\wedge} 3\right)$ for $\mathbf{1 0}$.

\begin{tabular}{lcccc}
\hline & \multicolumn{3}{c}{ z } \\
& & & \\
& & & \\
& & & \\
$\mathrm{H}(1)$ & 6044 & 5814 & 9496 & 25 \\
$\mathrm{H}(4)$ & 9435 & 4518 & 9548 & 26 \\
$\mathrm{H}(6)$ & 8952 & 3484 & 10569 & 33 \\
$\mathrm{H}(7)$ & 8422 & 2500 & 11616 & 39 \\
$\mathrm{H}(8)$ & 6619 & 3014 & 12250 & 40 \\
$\mathrm{H}(9)$ & 5356 & 4549 & 11832 & 36 \\
$\mathrm{H}(10)$ & 5855 & 5547 & 10784 & 30 \\
$\mathrm{H}(12)$ & 4971 & 7493 & 9739 & 34 \\
$\mathrm{H}(13)$ & 4818 & 9131 & 10444 & 37 \\
$\mathrm{H}(15)$ & 8601 & 8729 & 11303 & 35 \\
$\mathrm{H}(16)$ & 8758 & 7116 & 10579 & 31 \\
$\mathrm{H}(18 \mathrm{~A})$ & 8515 & 1389 & 8450 & 44 \\
$\mathrm{H}(18 \mathrm{~B})$ & 7136 & 1683 & 8769 & 44 \\
$\mathrm{H}(19 \mathrm{~A})$ & 9353 & 836 & 9672 & 79 \\
$\mathrm{H}(19 \mathrm{~B})$ & 8119 & 90 & 9364 & 79 \\
$\mathrm{H}(19 \mathrm{C})$ & 7933 & 1062 & 9956 & 79 \\
$\mathrm{H}(21 \mathrm{~A})$ & 9974 & 4428 & 6956 & 56 \\
$\mathrm{H}(21 \mathrm{~B})$ & 10718 & 3446 & 7426 & 56 \\
$\mathrm{H}(21 \mathrm{C})$ & 11269 & 4692 & 7501 & 56 \\
\hline
\end{tabular}




$\begin{array}{lllll}\mathrm{H}(23 \mathrm{~A}) & 5663 & 8603 & 7608 & 56 \\ \mathrm{H}(23 \mathrm{~B}) & 5334 & 7749 & 6933 & 56 \\ \mathrm{H}(23 \mathrm{C}) & 6784 & 8258 & 7084 & 56\end{array}$

